\title{
Chd7 Is Critical for Early T-Cell Development and Thymus Organogenesis in Zebrafish
}

\author{
Zhi-Zhi Liu, ${ }^{* \dagger \dagger}$ Zi-Long Wang, ${ }^{\star \S}$ Tae-Ik Choi, ${ }^{\oplus}$ Wen-Ting Huang, ${ }^{\dagger}$ Han-Tsing Wang, ${ }^{* \dagger}$ Ying-Ying Han, ${ }^{* \dagger}$ Lou-Yin Zhu, ${ }^{* \dagger}$

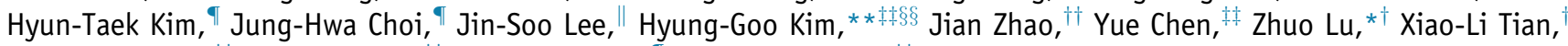 \\ Bing-Xing Pan, ${ }^{* \dagger}$ Bao-Ming Li, ${ }^{*}$ Cheol-Hee Kim, and Hong A. Xu ${ }^{* \dagger \ddagger}$
}

\begin{abstract}
From the Institute of Life Science, ${ }^{*}$ the School of Life Sciences, ${ }^{\dagger}$ the Queen Mary School,,$^{\S}$ and the Hospital of Nanchang University, ${ }^{\dagger \dagger}$ Nanchang University, Nanchang, China; the Children’s Hospital of Jiang Xi, ${ }^{\ddagger}$ Jiangxi Provincial Collaborative Innovation Center for Cardiovascular, Digestive and Neuropsychiatric Diseases, ${ }^{\ddagger}$ Nanchang, China; the Department of Biology, ${ }^{\complement}$ Chungnam National University, Daejeon, Republic of Korea; the National Cancer Center, "Goyang, Republic of Korea; and the Departments of Obstetrics and Gynecology, ${ }^{* *}$ and Neuroscience and Regenerative Medicine, ${ }^{\S \S}$ Augusta University, Augusta, Georgia
\end{abstract}

\author{
Accepted for publication \\ December 7, 2017. \\ Address correspondence to \\ Hong Xu, Ph.D., Institute of \\ Life Science, Nanchang Uni- \\ versity, Nanchang 330031, \\ China; or Cheol-Hee Kim, \\ Ph.D., Department of Biology, \\ Chungnam National University, \\ Daejeon 34134, Republic of \\ Korea. E-mail: xuhong@ncu. \\ edu.cn or zebrakim@cnu.ac.kr.
}

\begin{abstract}
Coloboma, heart defect, atresia choanae, retarded growth and development, genital hypoplasia, ear anomalies/deafness (CHARGE) syndrome is a congenital disorder affecting multiple organs and mainly caused by mutations in $\mathrm{CHD7}$, a gene encoding a chromatin-remodeling protein. Immunodeficiency and reduced T cells have been noted in CHARGE syndrome. However, the mechanisms underlying T lymphopenia are largely unexplored. Herein, we observed dramatic decrease of T cells in both chd7knockdown and knockout zebrafish embryos. Unexpectedly, hematopoietic stem and progenitor cells and, particularly, lymphoid progenitor cells were increased peripherally in nonthymic areas in chd7-deficient embryos, unlikely to contribute to the T-cell decrease. Further analysis demonstrated that both the organogenesis and homing function of the thymus were seriously impaired. Chd7 might regulate thymus organogenesis through modulating the development of both neural crest cell-derived mesenchyme and pharyngeal endoderm-derived thymic epithelial cells. The expression of foxn1, a central regulator of thymic epithelium, was remarkably down-regulated in the pharyngeal region in chd7-deficient embryos. Moreover, the T-cell reduction in chd7-deficient embryos was partially rescued by overexpressing foxn1, suggesting that restoring thymic epithelium may be a potential therapeutic strategy for treating immunodeficiency in CHARGE syndrome. Collectively, the results indicated that chd7 was critical for thymic development and T-lymphopenia in CHARGE syndrome may be mainly attributed to the defects of thymic organogenesis. The current finding may benefit the diagnosis and therapy of T lymphopenia and immunodeficiency in CHARGE syndrome. (Am J Pathol 2018, 188: 1043-1058; https://doi.org/10.1016/ j.ajpath.2017.12.005)
\end{abstract}

Coloboma, heart defect, atresia choanae, retarded growth and development, genital hypoplasia, ear anomalies/deafness (CHARGE) syndrome (Online Mendelian Inheritance in Man no. 214800) is a complex genetic developmental disorder featuring a unique combination of multiple organ anomalies, including coloboma, heart defects, atresia of the choanae, retarded growth and development, genital hypoplasia, and ear anomalies. ${ }^{1,2}$ Additional features have been recognized in CHARGE syndrome since its first description, such as cranial nerve defects, anosmia, and renal and skeletal abnormalities. ${ }^{3-6}$ Mutations within the chromodomain helicase DNA binding protein 7 (CHD7) gene are the major known cause of CHARGE syndrome, accounting for the disease in at least $60 \%$ of affected individuals. $^{2,7}$

\footnotetext{
Supported by the National Natural Science Foundation of China grants 31400988 (Z.-Z.L.), 81160144 (H.X.), and 31171044 (H.X.), Young Scientist of Jiangxi Province grant 20122BCB23007 (H.X.), the Natural Science Foundation of Jiangxi Province grant 20151BAB215015 (Z.Z.L.), and the National Cancer Center grant NCC1210360 (C.H.K.).

Disclosures: None declared.
} 
CHARGE patients usually experience recurrent infections during infancy, and these infections are presumed to result from anatomic anomalies of the craniofacial region and upper airway and defects of the innervating cranial nerves. The contribution of immunodeficiency to these infections has been noted in some case reports, but has received only limited attention. ${ }^{8-11}$ Recent clinical studies suggest that immunodeficiency is more commonly involved than previously thought. For instance, Wong et $\mathrm{al}^{12}$ reviewed immunodeficiency in CHARGE syndrome and found that $80 \%$ of the patients had T lymphopenia. Furthermore, $\mathrm{CHD7}$ is recognized as a susceptibility gene for severe combined immunodeficiency in newborn screening studies. ${ }^{13,14}$ Thymic aplasia or hypoplasia has been reported in patients with CHARGE syndrome. However, thymus has not been routinely examined in CHARGE patients and the rate of thymic defects varied dramatically in different reports. ${ }^{12,15}$ The current understanding of immunodeficiency, T-cell reduction, and thymus defects in CHARGE syndrome is largely limited to clinical observations and the underlying pathogenic mechanisms are largely unexplored experimentally.

In this study, we investigated the role of $c h d 7$ in T-cell and thymic development using zebrafish as a model organism. Knockdown or deletion of chd7 decreased hematopoietic stem and progenitor cell (HSPC) numbers in the thymus but increased those numbers in peripheral nonthymic regions of zebrafish embryos. We revealed that chd7 was critical for thymic organogenesis through regulating both thymic epithelial cells (TECs) and neural crest cells (NCCs). Moreover, T-cell deficiency may be partially rescued by forced expression of foxn1. Last, we suggested a molecular pathway regulated by $\operatorname{chd} 7$ that is crucial for thymic organogenesis. These results suggested the involvement of thymic dysplasia and $\mathrm{T}$ lymphopenia in immunodeficiency in CHARGE syndrome.

\section{Materials and Methods}

\section{Fish Strains and Embryos}

Zebrafish strains, including wild type (AB strain), chd7 mutant, rag2:enhanced green fluorescence protein (EGFP), lyz:DsRed, and corola:EGFP transgenic lines, were purchased from the China Zebrafish Resource Center (Wuhan, China). Embryos were obtained by natural spawning and raised in E3 buffer at $28^{\circ} \mathrm{C}$. Adult zebrafish were raised in a zebrafish breeding system (HAISHENG Biotech, Shanghai, China) at $28^{\circ} \mathrm{C}$. This study was approved by the Ethical Review Committee of Nanchang University (Nanchang, China).

\section{Morpholinos, Primers, mRNA Synthesis, and Microinjection}

Standard morpholino (MO) and antisense MO were purchased from GeneTools (Philomath, OR) and prepared as $1 \mathrm{mmol} / \mathrm{L}$ stock solutions using double-distilled water (Millipore,
Burlington, MA). The sequences of MOs and the primers used for amplification of RNA probes and coding sequences are listed in Table 1. Capped zebrafish full-length foxn1 mRNA for injection was synthesized in vitro using the mMessage mMachine SP6 transcription kit (Ambion, Carlsbad, CA). MOs (8 ng for $c h d 7 \mathrm{MO}$ and standard MO) and capped mRNA $(100 \mathrm{pg})$ were injected alone or together into one cell stage zebrafish embryos at the yolk/blastomere boundary.

\section{PCR}

All primers used for the preparation of whole mount in situ hybridization (WISH) probes, RT-PCR, and real-time quantitative PCR (qPCR) are listed in Table 1. Total RNA was extracted by grinding dechorionated 15 to 20 embryos using RNAiso plus (Takara, Shiga, Japan) and followed by phenol-chloroform extraction. Reverse-transcription reaction was performed by using M-MLV Reverse Transcriptase (Takara). Wild-type and morphant chd7 transcripts were detected using RT-PCRs. qPCR was performed using the SYBR Premix Ex Taq II (Takara) on the Abi-Step-One plus Real-Time PCR system (Applied Biosystems, Foster City, CA). All of the experiments were repeated three times with triplicates. The data were analyzed using the $\triangle \triangle \mathrm{Ct}$ method. Data were represented as means $\pm \mathrm{SD}$, and $t$-test was performed for comparison between control and injected groups. $P<0.05$ indicates significant difference.

\section{Whole-Mount in Situ Hybridization}

WISH was performed, as previously described, ${ }^{16}$ using probes for chd7, rag1, il7r, cmyb, ikaros, mfap4, mpo, foxn1, ccl25a, and $c c l 25 b$. Briefly, staged embryos were fixed overnight in $4 \%$ paraformaldehyde, and then dehydrated in a methanol gradient. When ready to use, embryos were rehydrated in phosphatebuffered saline containing $0.1 \%$ Tween-20. Embryos were permeabilized by proteinase $\mathrm{K}$ digestion and then hybridized with digoxin-labeled probes overnight at $70^{\circ} \mathrm{C}$. The next day, embryos were washed in a preheated mixture of $50 \%$ saline-sodium citrate containing $0.1 \%$ Tween-20 and $50 \%$ hybridization solution at $70^{\circ} \mathrm{C}$. Embryos were washed again at room temperature and incubated in staining solution in the dark until sufficient staining appeared in the embryos. Background staining was cleared by soaking the embryos in a benzylbenzoate-benzylalcohol solution. Embryos were mounted in glycerol and were visualized using a Nikon AZ100 microscope (Nikon, Tokyo, Japan). Images were captured using a Nikon DIGITAL SIGHT DS-Fill digital camera (Nikon) and processed with NIS-Elements F 3.0 (Nikon).

\section{Whole-Mount Immunostaining}

For immunofluorescence microscopy, immunostaining was performed as described previously. ${ }^{17}$ Larval zebrafish were fixed overnight with $4 \%$ paraformaldehyde in PBS, followed by methanol dehydration and rehydration. After 
Table 1 Sequences of the MO and Primers Used in this Study

\begin{tabular}{|c|c|c|}
\hline Name & Sequence & Use \\
\hline Chd7 M0 & 5'-TTATTTTCTGGCACTAACCATGTCC-3' & Gene knockdown \\
\hline Chd7 ATG-M0 & $5^{\prime}-$ TGCAGCCAAGCTTAGAAGCAGGAC- $3^{\prime}$ & \\
\hline ikaros- $\mathrm{F}$ & 5'-TTCCAGTGCAATCAATGTGGTGCTT-3' & $q P C R$ \\
\hline ikaros-R & 5'-GCACACTTATGGGGCTTTCCAACCG-3' & \\
\hline $\operatorname{rag} 1-\mathrm{F}$ & 5'-TAGGAAAGAAGCGGACTAAACCACT-3' & qPCR \\
\hline $\operatorname{rag} 1-\mathrm{R}$ & 5'-GCACCGTGTGATATTCTTTACCCACT-3' & \\
\hline$c c l 25 b-\mathrm{F}$ & 5'-TTGTATGGAAACGCAGAGTTCAACC-3' & qPCR \\
\hline$c c l 25 b-\mathrm{R}$ & 5'-CTCATCTTGAAAACAACAGCGGGAA-3' & \\
\hline$i l 7 r-\mathrm{F}$ & $5^{\prime}$-TGCAAGTACACTTAAGAGACGGCAT-3' & $q P C R$ \\
\hline$i l 7 r-\mathrm{R}$ & 5'-GCTCATGTTCACCACGGATCTCCA-3' & \\
\hline foxn1-F & 5'-TTTAAATAGCGTGAATTCTATGCTG-3' & qPCR \\
\hline foxn1-R & $5^{\prime}$-CTACCGCTTCACATGAACGAG-3' & \\
\hline elf1a-R & 5'-GGAGCGGTGAGTTTGAGGCTGGTATCTCC-3' & \\
\hline ikaros-F & 5'-TTGGAAAGCCCCATAAGTGTGCAT-3' & WISH probe \\
\hline ikaros-R & 5'-GTACCCGCATAGGTTGCACTCA-3' & \\
\hline $\operatorname{rag} 1-\mathrm{F}$ & 5'-AGACTGGAAGCCCCACACCTCT-3' & WISH probe \\
\hline $\operatorname{rag} 1-\mathrm{R}$ & $5^{\prime}$-CCTCTGGCACCGTGTGATATTC-3' & \\
\hline hand2-F & 5'-АCCTCATTGATTCCACAACGTGCTC-3' & WISH probe \\
\hline hand2-R & 5'-TAAGCGATATAACTGGTAGCTAAACGA-3' & \\
\hline$N k \times 2.3-\mathrm{F}$ & $5^{\prime}$-AGTCCCGGCTTCTCAGACAACGA-3' & WISH probe \\
\hline$N k \times 2.3-R$ & 5'-TTCAGCCATCATGGTATACTGTTGC-3' & \\
\hline$d l \times 2 a-F$ & $5^{\prime}$-GCCAAAGAAAGTCCGAAAACCTCGT-3' & WISH probe \\
\hline$d l \times 2 a-R$ & 5'-TCAGGTTATTTTCAAGAAGGCACCA-3' & \\
\hline
\end{tabular}

ATG, translation-blocking; Chd, chromodomain helicase DNA binding protein; F, forward; M0, morpholino; $q P C R$, real-time quantitative PCR; R, reverse; WISH, whole mount in situ hybridization.

permeabilization by ice-cold acetone treatment and proteinase $\mathrm{K}$ digestion, embryos were incubated with primary and secondary antibodies at the following concentrations: 1:500 mouse anti-alcama (Zirc Co, Buffalo, MN), 1:500 goat anti-GFP (Rockland, Limerick, PA), 1:500 antigoat IgG Alexa Fluor 488 (Invitrogen, Carlsbad, CA), and 1:500 anti-mouse IgG (Invitrogen). Immunostained embryos were imaged using an Olympus FV1000 confocal microscope (Olympus, Tokyo, Japan).

\section{Terminal dUTP Nick End Labeling Assay}

Wild-type or corola:EGFP transgenic embryos were fixed in $4 \%$ paraformaldehyde at $4{ }^{\circ} \mathrm{C}$ overnight and then dehydrated in a methanol gradient and preserved in $100 \%$ methanol at $-20^{\circ} \mathrm{C}$ overnight. After rehydration, the embryos were washed with phosphate-buffered saline (PBS) containing $0.1 \%$ Tween-20 three times and treated with $5 \mu \mathrm{g} / \mathrm{mL}$ proteinase $\mathrm{K}$ for permeabilization. Then, the permeabilized embryos were refixed in $4 \%$ paraformaldehyde for 30 minutes at room temperature with gentle shaking. After being washed three to five times with PBS containing $0.1 \%$ Tween20 , the embryos were incubated with a mixture containing 45 $\mu \mathrm{L}$ labeling solution and $5 \mu \mathrm{L}$ enzyme solution (In Situ Cell Death Detection Kit Fluorescein; Roche, Basel, Switzerland) at $4^{\circ} \mathrm{C}$ overnight. Finally, the stained embryos were washed three times with PBS containing $0.1 \%$ Tween-20 and imaged using an Olympus FV1000 confocal microscope.

\section{Confocal Microscopy}

Live embryos were anesthetized with tricaine and then mounted onto a coverslip with $1 \%$ low-melting agarose. The agar layer was further covered with E3 buffer containing $0.0084 \mathrm{mg} / \mathrm{mL}$ tricaine to enable movies to be taken over a long time frame. For live imaging of the thymus, the recordings were started at 60 hours post-fertilization (hpf) and continued for 12 hours. Images were taken every 6 or 5 
A

chd7 expression pattern
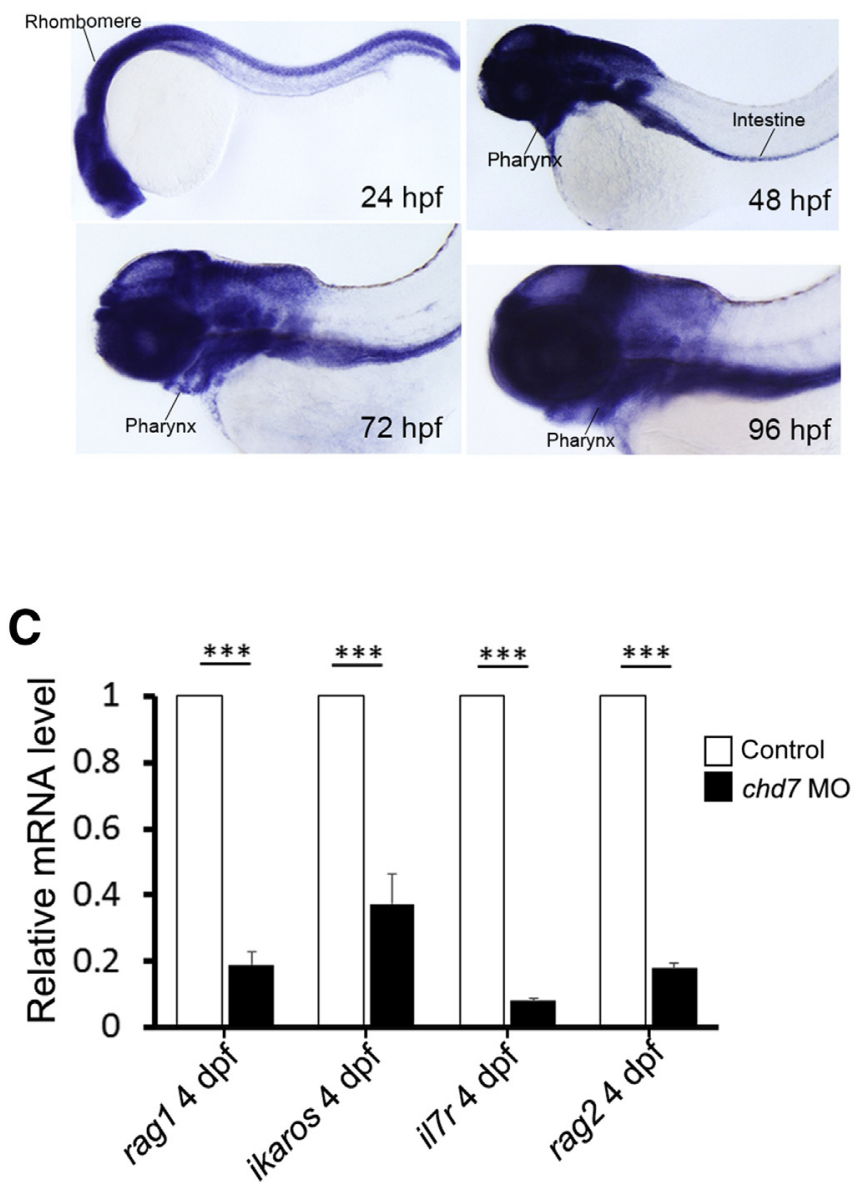

B

\section{Control}

chd7 $\mathrm{MO}$

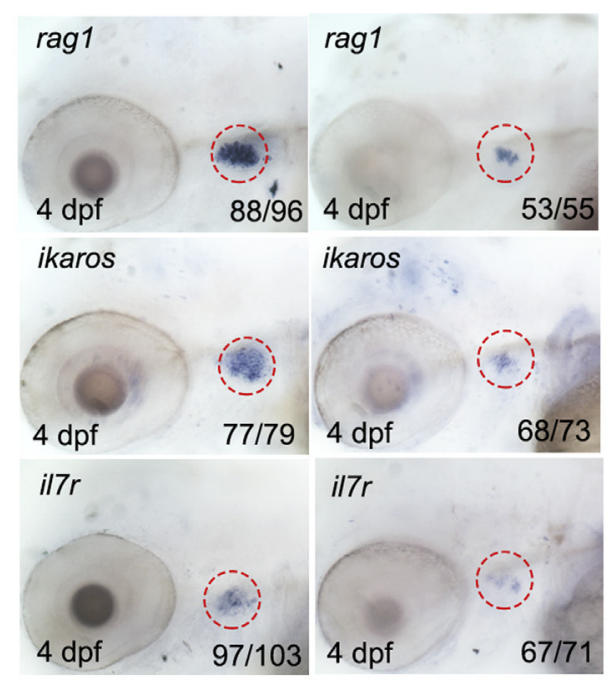

D chd7 $\mathrm{MO}$

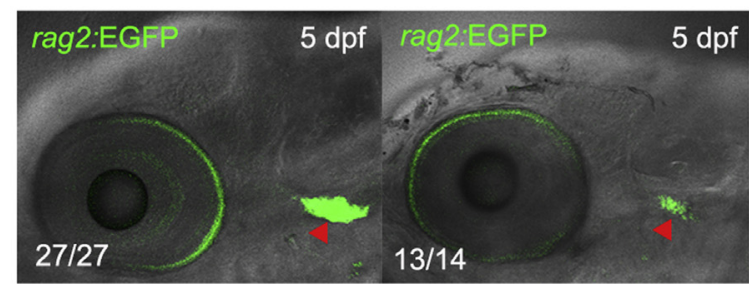

Figure 1 T-cell development is impaired in chd7 zebrafish morphants. A: The expression pattern of endogenous chd7 transcripts at different time points. B: The expression of T-cell markers, including rag1, ikaros, and $i l 7 r$, in control and chd7 morphants. Anterior to the left and dorsal to the top, red dashed circles mark the thymus. C: Quantitative RT-PCR analysis showing the mRNA level of rag1, ikaros, and il7r in control and chd7 morphants ( $t$-test). D: rag2:Enhanced green fluorescence protein (EGFP) expression in control and chd7 morphants at 5 days post-fertilization (dpf). The red arrowheads indicate the rag2:EGFP expression in thymus. Data are expressed as means \pm SD. ${ }^{* *} P<0.001$. Hpf, hours post-fertilization; M0, morpholino. Original magnification: $\times 3$ (A, 24 hpf, $48 \mathrm{hpf}$, and $72 \mathrm{hpf}) ; \times 6$ (A, $96 \mathrm{hpf}) ; \times 15$ (B); $\times 20$ (D).

minutes, and single frames were generated as projections of a stack of 46 planes with $2-\mu \mathrm{m}$ distance, covering a depth of $90 \mu \mathrm{m}$. For imaging immunostained samples, embryos were mounted onto a coverslip with $1 \%$ low-melting agarose. Confocal images were captured by an Olympus FV1000 confocal microscope and three-dimensional projections were generated with the FV100-ASW 4.2 viewer (Olympus). Single frames were generated as projections of a stack of multiple planes. The numbers of immunofluorescence- and terminal dUTP nick end labeling cells were calculated using Imaris7 (Bitplane, Zürich, Switzerland).

\section{Alcian Blue Staining}

Zebrafish larvae [5 days post-fertilization (dpf)] were fixed in $4 \%$ paraformaldehyde at $4{ }^{\circ} \mathrm{C}$ overnight. Then, the fixed larvae were digested in $10 \%$ trypsin for 1 hour. After washing in PBS three times, the larvae were stained in $0.1 \%$ (w/v) Alcian blue (Sigma, Darmstad, Germany) for 3 hours. Then, the staining reaction was terminated with PBS, and the larvae were washed again in PBS. The stained larvae were mounted in $90 \%$ glycerol and visualized using a Nikon AZ100 microscope. Images were captured using a Nikon DIGITAL SIGHT DS-Fill digital camera and processed with NIS-Elements F 3.0 software (Nikon).

\section{Statistical Analysis}

Values are presented as the means (SD). $t$-Test was used to compare means of different groups, and $P<0.05$ was considered significant. 
A

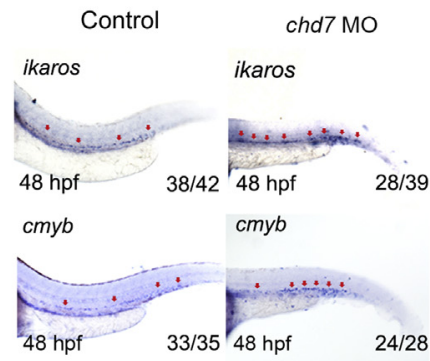

C

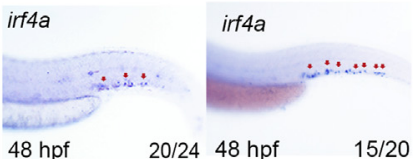

$\mathbf{E}$

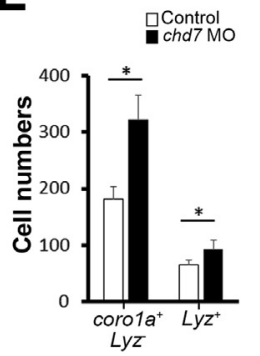

$\mathbf{F}$

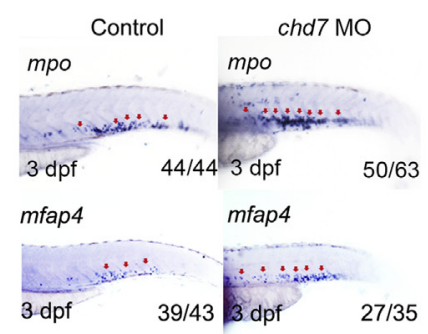

H

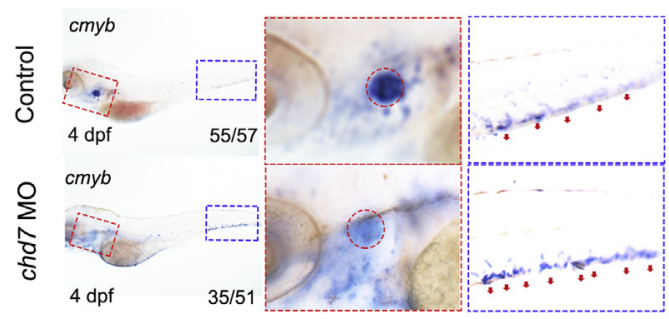

\section{B}

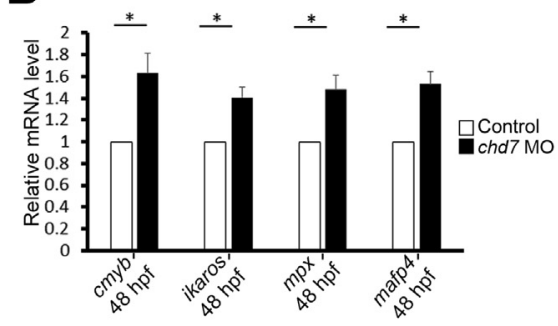

D

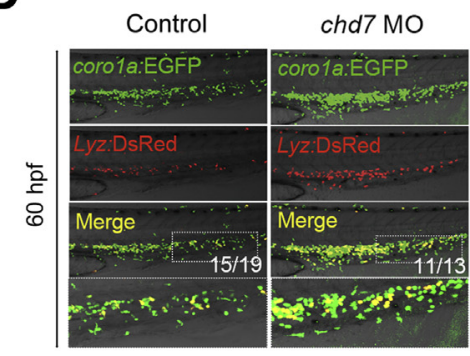

G

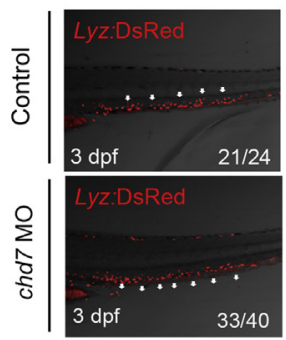

Figure 2 Hematopoietic stem and progenitor cell numbers are reduced in the thymus but increased in the caudal hematopoietic tissue (CHT) region in chd7 morphants. A: The expression of hematopoietic progenitor markers, ikaros and cmyb, in control and chd7 morphants at 48 hours post-fertilization (hpf). Red arrows denote hematopoietic progenitors expressing ikaros or cmyb in the aorta-gonad-mesonephros region. B: Quantitative RT-PCR analysis showing the mRNAs of cmyb, ikaros, mpo, and mafp 4 in control and chd7 morphant at $48 \mathrm{hpf}$ ( $t$-test). C: The expression of Tlymphoid primed progenitor marker, irf $4 a$, in control and chd7 morphant at 48 hpf. Red arrows indicate the T-lymphoid primed cells expressing irf4a in the CHT region. D: Double transgenic [coro1a:enhanced green fluorescence protein (EGFP)/lyz:DsRed] wild-type and chd7 morpholino (MO)-injected embryos showing the numbers of lymphoid progenitors, macrophages, and neutrophils in the CHT region of control and chd7 morphants at $60 \mathrm{hpf}$. White dashed rectangles indicate the posterior part of the $\mathrm{CHT}$ region. E: Enumeration of coro1a:EGFP-positive and lyz:DsRed-negative cells or lyz:DsRed cells shown in D. F: The expression of mpo and mfap4, which mark neutrophils and macrophages, respectively, in the CHT region of control and chd7 morphants at $3 \mathrm{dpf}$. Red arrows denote neutrophils expressing mpo or macrophages expressing mfap 4 in the CHT region. G: Neutrophils marked by lyz:DsRed expression in the $\mathrm{CHT}$ region of control and chd7 morphants at 3 days post-fertilization (dpf). White arrows indicate the DsRed-positive neutrophils. H: The expression of cmyb in control and chd7 morphants at $4 \mathrm{dpf}$. Red dashed rectangles denote the pharyngeal regions; blue dashed rectangles, the $\mathrm{CHT}$ regions; red dashed circles, the region of thymus; and red arrows, the hematopoietic stem progenitor cells and myeloid lineage cells in the CHT region. Data are expressed as means $\pm \mathrm{SD} .{ }^{*} P<0.05$. Original magnification: $\times 9(\mathbf{A}, \mathbf{C}$, and $\mathbf{F}) ; \times 10(\mathbf{D}$ and $\mathbf{G}) ; \times 1.8(\mathbf{H})$.

\section{Results}

\section{T-Cell Development Is Impaired in chd7-Deficient Embryos}

Embryonic $\mathrm{T}$ lymphopoiesis consists of several successive steps, and the process is evolutionarily conserved between zebrafish and higher vertebrates. ${ }^{18}$ In zebrafish, at around $48 \mathrm{hpf}$, the endodermal progenitors in the third pharyngeal pouch begin to differentiate into TECs, marked by the expression of tissuespecific transcription factors, foxnl. Subsequently, by interacting with postmigratory neural crest cells derived from the dorsal ectoderm, the thymic anlage detaches from the pharynx and moves into a location underneath the otic vesicles. Meanwhile, lymphoid progenitors in the aorta-gonad-mesonephros and caudal hematopoietic tissue (CHT) regions migrate into the thymic rudiment and become established for subsequent differentiation and maturation, and then $\mathrm{T}$ lymphopoiesis is initiated after the expression of ragl at $78 \mathrm{hpf}^{19}$

WISH was performed in zebrafish embryos, and it was found that $\operatorname{chd} 7$ was mainly expressed in central nervous system and gut (Figure 1A). It could be detected in rhombomeres at $24 \mathrm{hpf}$, from which some neural crest cells are derived (Figure 1A). In the developing pharynx from 48 to $96 \mathrm{hpf}$, chd7 was also strongly expressed (Figure 1A). These WISH data indicated that $c h d 7$ might participate in multiple aspects of embryonic development, including neural crest development and pharyngeal segmentation, which are related to thymic development.

An antisense oligonucleotide MO targeting the junction between exon 13 and intron 13 of $c h d 7$ to block splicing ${ }^{20}$ 
A
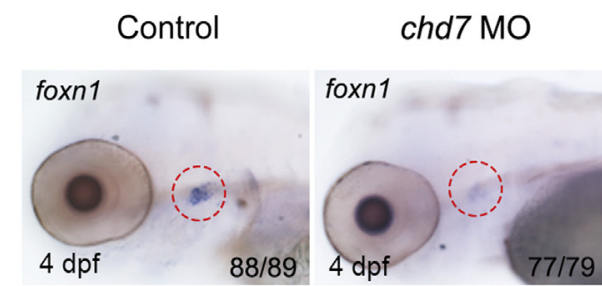

C

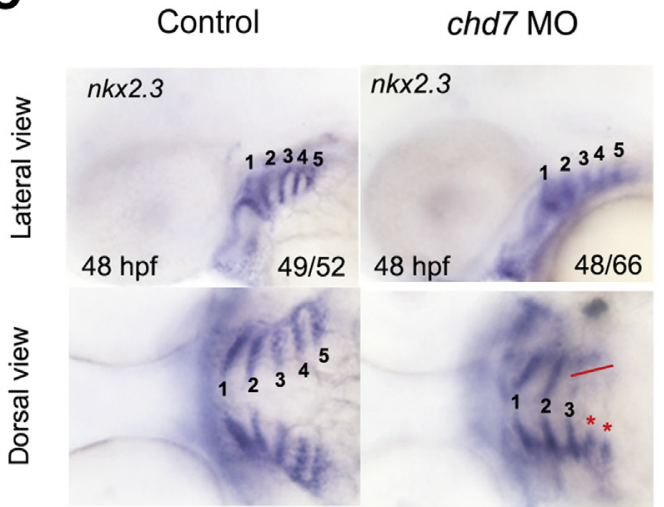

B

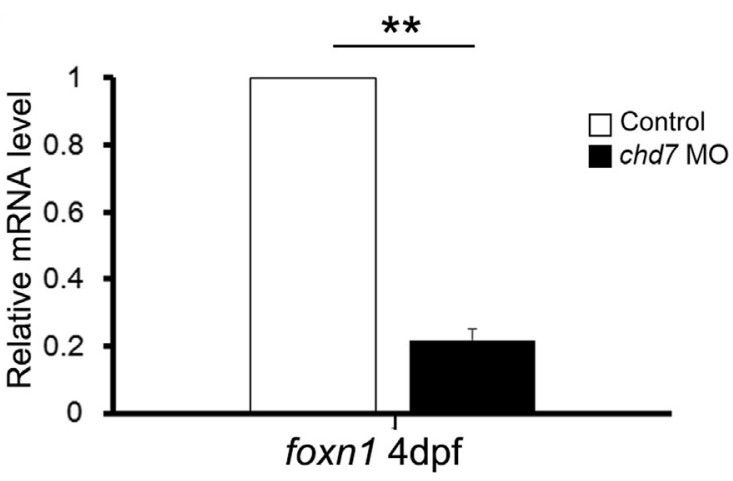

D

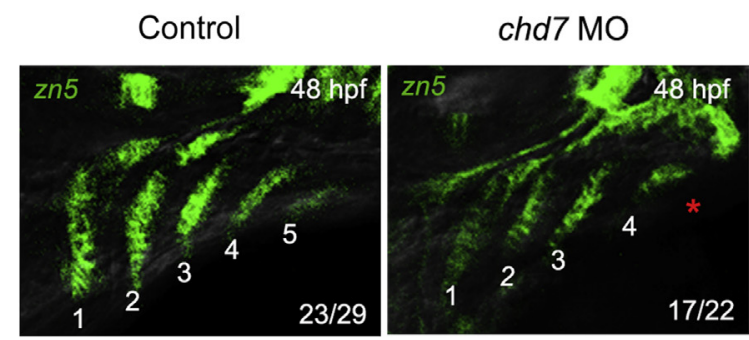

Figure 3 Thymic epithelium development is severely impaired in chd7 morphants. A: The expression of a thymic marker, foxn1, in the thymus of control and chd7 morphants. Red dashed circles mark the thymus. B: Quantitative RT-PCR showing the mRNA level of foxn1 in control and chd7 morphants at 4 days post-fertilization (dpf; $t$-test). C: The morphology of endodermal pouches shown by expression of $n k x 2.3$, in control and chd7 morphants at 48 hours postfertilization (hpf). Numbers indicate the pharyngeal pouches, and red asterisks and red line indicate the moderate and severe defects, respectively, in endodermal pouches 3, 4, and 5. D: Immunohistochemistry with zn5 showing the morphology of endodermal pouches in chd7 morphants at 48 hpf. Numbers denote the pharyngeal pouches, and red asterisk indicates the absence of fifth pharyngeal pouch. Data are expressed as means \pm SD. ${ }^{* * P}<0.01$. M0, morpholino. Original magnification: $\times 12(\mathbf{A}) ; \times 15$ (C); $\times 20$ (D).

(Table 1) was injected into zebrafish zygotes. Sequencing the RT-PCR product revealed that intron 13 was retained in the transcript, introducing a premature stop codon at the 1175th codon and presumably resulting in a truncated protein missing SNF2, helicase, and BRK domains (Supplemental Figure S1). Several CHARGE-like phenotypes were observed among the chd7 morphants, including coloboma, abnormal otoliths, cartilage defects, and reversed heart looping (Supplemental Figure S2), which were consistent with a previous report. ${ }^{20}$

To explore the role of $c h d 7$ in T lymphopoiesis, WISH was performed to check the expression of several T-cell markers, including ragl, ikaros, and il7r. Compared with control, the expression of all these markers was dramatically reduced in the thymus at $4 \mathrm{dpf}$ (Figure 1B). Real-time quantitative PCR further confirmed the WISH observations that T-cell markers were significantly down-regulated in 4-dpf morphants (Figure 1C). Moreover, when $c h d 7 \mathrm{MO}$ was injected into a rag2:EGFP transgenic fish line at the single cell stage, the number of GFP-positive T-cell precursors was significantly decreased at $5 \mathrm{dpf}$ (Figure 1D). Taken together, these results indicated that knockdown of $c h d 7$ may model the T lymphopenia found in CHARGE patients.
Hematopoietic Progenitor and T-Lymphoid Primed Progenitor Numbers Are Increased in the Nonthymic Region of chd7 Morphants

The reduction of $\mathrm{T}$ cells may be attributable to the decrease of HSPCs, the defects of thymus organogenesis, or abnormal proliferation or apoptosis of thymocytes. The development of HSPCs was first studied. Surprisingly, HSPCs were increased in the aorta-gonad-mesonephros and CHT regions in $c h d 7$ morphants at $48 \mathrm{hpf}$, as indicated by the expression of cmyb and ikaros, two HSPC markers at the prethymic stage (Figure 2A). qPCR data further confirmed the WISH findings (Figure 2B). To further address the development of lymphoid progenitors, the expression of irf4a, a recently identified marker of $\mathrm{T}$ lymphoid primed progenitors, ${ }^{21}$ was examined, and it was also found to be increased in the CHT region at $50 \mathrm{hpf}$ in chd7 morphants (Figure 2C). Because irf4a is only expressed in a small population of T-lymphoid-primed progenitors, we used corola:EGFP/lyz:DsRed double transgenic embryos to mark the lymphoid progenitors and macrophages (corola:EGFP expression marks both myeloid and lymphoid lineages, and lyz:DsRed expression 
A
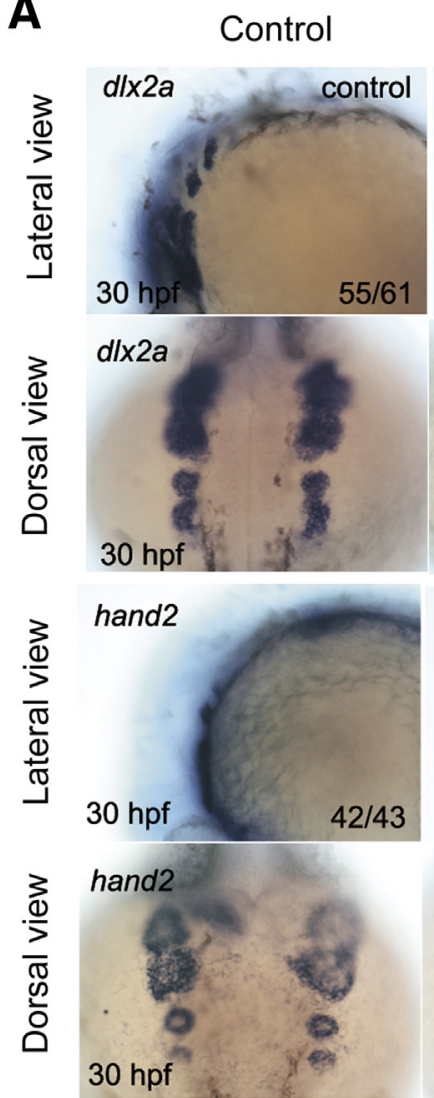

chd7 MO
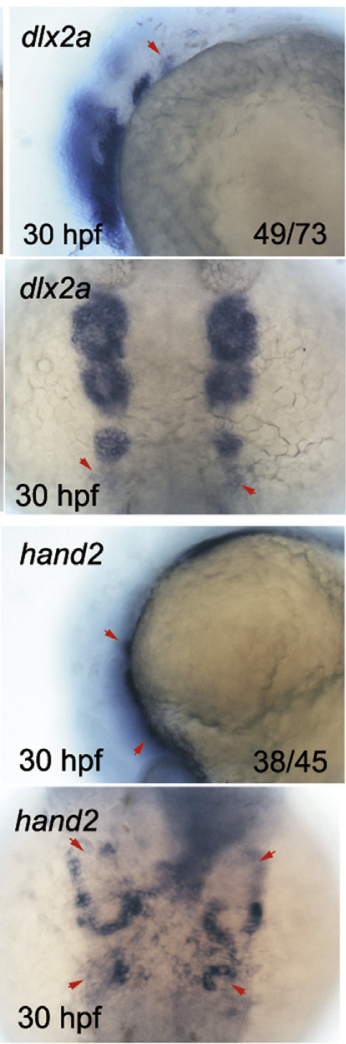

B

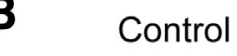

chd7 $\mathrm{MO}$
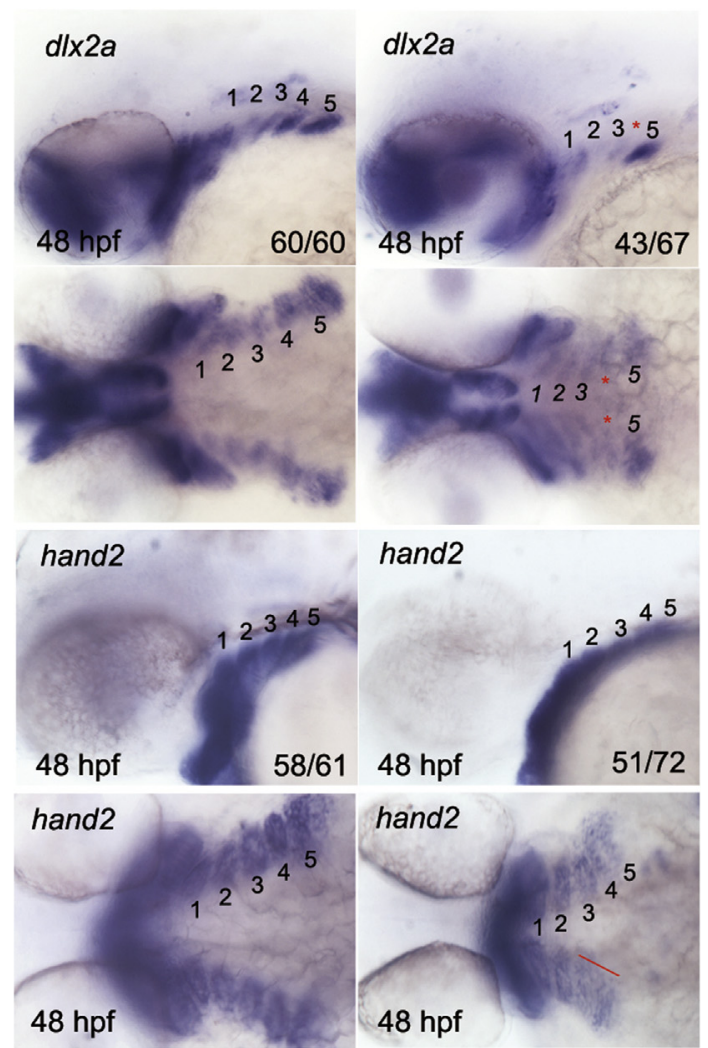

hand2

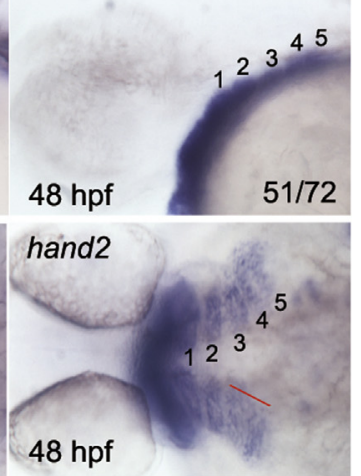

Figure 4 The development of post-migratory neural crest cells is impaired in chd7 morphants. A: The expression of post-migratory neural crest cells markers, $d \times 2 a$ and hand2, in pharyngeal arches in control and chd7 morphants at 30 hours post-fertilization (hpf). Red arrows indicate the reduced postmigratory neural crest cells. B: The expression of post-migratory neural crest cells markers, $d \times 2 a$ and hand2, in pharyngeal arches in control and chd7 morphants at $48 \mathrm{hpf}$. Numbers denote the pharyngeal arches; red asterisks, the defective pharyngeal arches; red line, the obscure pharyngeal arches. M0, morpholino. Original magnification: $\times 9(\mathbf{A}$, lateral views); $\times 12(\mathbf{A}$, dorsal views, and $\mathbf{B})$.

marks neutrophils). ${ }^{22}$ The GFP-positive but DsRed-negative cells (lymphoid progenitors and macrophages) were modestly increased in the CHT region on chd7 knockdown (Figure 2, D and E). These results implied that the T-cell deficiency in $\operatorname{ch} d 7$ morphants was unlikely to be attributed to a lack of T-lymphoid-primed progenitors at prethymic stage. It was further tested whether the HSPCs and myeloid lineages were changed when thymus rudiment was formed. The expression of mpo and mfap4, which mark neutrophils and macrophages, respectively, was first examined. Both WISH and qPCR data showed that mpo and mfap4 were increased in the CHT region at $3 \mathrm{dpf}$ (Figure 2, B and F). Moreover, when the chd7 MO was injected into Lyz:DsRed transgenic embryos, the DsRed-positive neutrophils were increased at $3 \mathrm{dpf}$ (Figure 2G). Furthermore, to overview the distribution of hematopoietic cells in chd7 morphants, the expression of $c m y b$, which was expressed in HSPCs and myeloid lineage cells, was examined. The expression of $c m y b$ was specifically reduced in the thymus, whereas it was moderately increased in the CHT region of $c h d 7$ morphants at $4 \mathrm{dpf}$ (Figure 2H). Analogously, when the $c h d 7 \mathrm{MO}$ was injected into corola:EGFP transgenic embryos, a specific reduction of EGFP-positive $\mathrm{T}$ cells was detected in the thymus at $4 \mathrm{dpf}$ (Supplemental Figure S3). Collectively, our data demonstrated that HSPCs were specifically reduced in the thymic region but increased in the nonthymic region, suggesting that the T-cell defect in $c h d 7$ morphants seemed unlikely to be explained by the increase of T-lymphoid progenitors. Alternatively, it may be caused by the defects of other T-cell development processes (eg, thymic organogenesis).

The Development of Thymic Epithelial Cells Is Severely Impaired in chd7 Morphants

TECs are the primary functional cells that form the microenvironment to support T-cell differentiation. FOXN1 is a marker of TECs and a pivotal regulator of TEC differentiation, proliferation, and survival ${ }^{23}$; therefore, its expression was examined. Both WISH and qPCR data showed a dramatic reduction of foxnl expression in the thymus of chd7 morphants at $4 \mathrm{dpf}$ (Figure 3, A and B). Because both the cortical and medullary TECs might arise from the single endodermal layer in the third pharyngeal pouch, ${ }^{24}$ it was 
A
A Control

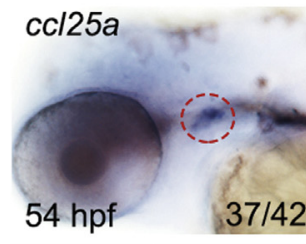

ccl25b

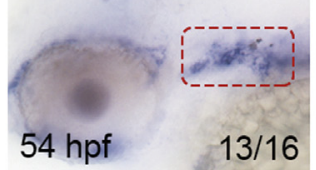

chd7 $\mathrm{MO}$

ccl25a

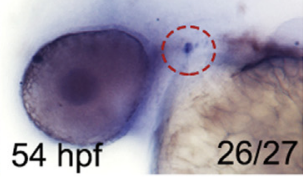

cc/25b

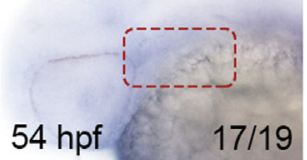

B

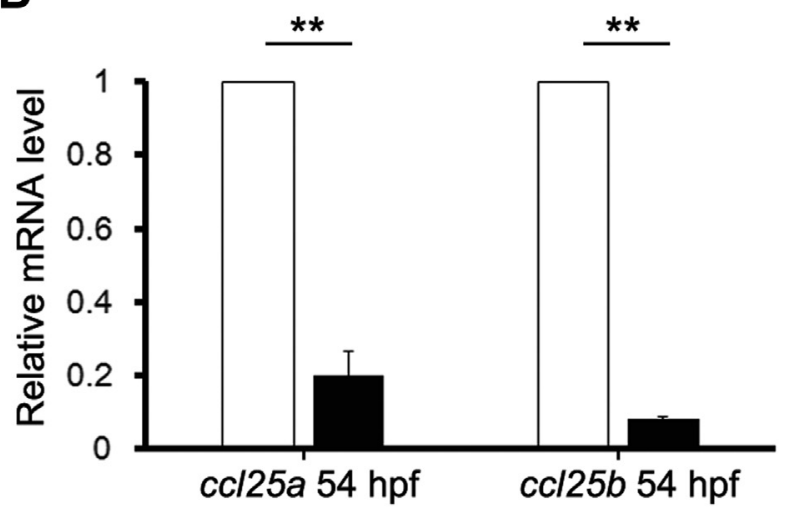

D

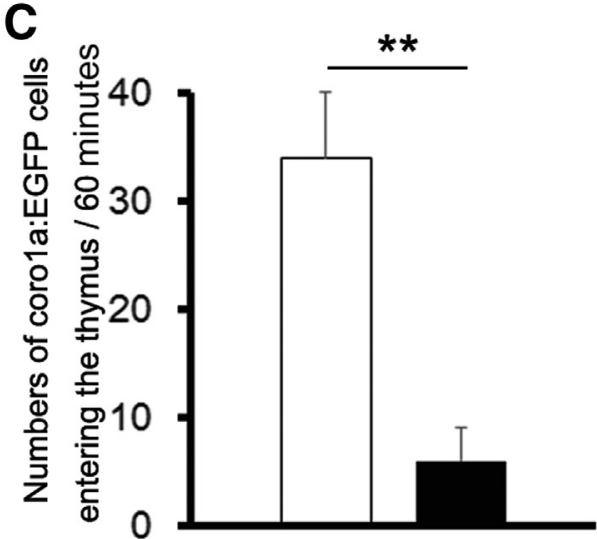

coro1a:EGFP/lyz:DsRed (60 hpf)

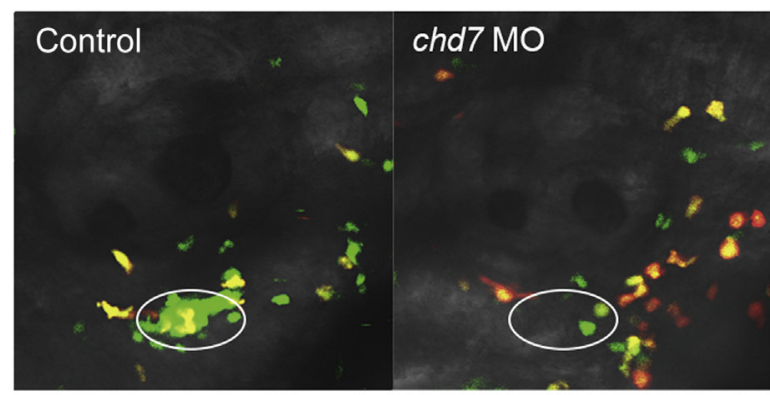

Figure 5 The thymic homing is disrupted and chemokine ligands are down-regulated in chd7 morphants. A: Whole mount in situ hybridization for ccl25a and $c c l 25 b$ in the control and chd7 morphants at 54 hours post-fertilization (hpf). Red dashed circles and rectangles indicate the thymic regions. B: Real-time quantitative PCR analysis of mRNA level of $c c l 25 a$ and $c c l 25 b$ in the control and chd7 morphants at $54 \mathrm{hpf}(t$-test). C: Number of coro1a:enhanced green fluorescence protein (EGFP) cells entering the thymus every 60 minutes in the control and chd7 morphants during the homing process from 60 to 72 hpf. D: Confocal imaging of coro1a:EGFP/lyz:DsRed embryos showing the numbers of coro1a:EGFP cells in the thymus in control and chd7 morphants. White circles indicate the thymus. Data are expressed as means \pm SD. $n=3$ (C). ${ }^{* *} P<0.01$. Original magnification: $\times 9$ (A); $\times 60$ (B). M0, morpholino.

tested whether the development of pharyngeal pouches was impaired in $c h d 7$ morphants. To address this, the expression of $n k x 2.3$, a homeobox transcription factor that is expressed in the endoderm of developing pharyngeal pouches in vertebrates, was examined. ${ }^{25}$ The expression of $n k x 2.3$ in chd7 morphants was greatly decreased in pouches 3,4 , and 5 , and the whole pharynx appeared shrunken (Figure 3C). To further corroborate this result, immunohistochemistry was performed with antibody zn5, which recognizes activated leukocyte cell adhesion molecule expressed in pharyngeal endoderm. ${ }^{26,27}$ The immunofluorescence in all five pharyngeal pouches was decreased, indicating that the development of these pouches was disrupted in $c h d 7$ morphants (Figure 3D). Taken together, these results indicated that both morphogenesis of pharyngeal endoderm and TEC differentiation were disrupted in $c h d 7$ morphants.

The Development of Post-Migratory Neural Crest Cells Is Impaired in Pharyngeal Arches in chd7 Morphants

NCCs are a transient population of pluripotent cells arising from the dorsal ectoderm and are required for thymus development. ${ }^{28-30}$ CHD7 has been characterized as a critical regulator in migratory and multipotent neural crest cells. ${ }^{31}$ Therefore, it is reasonable to speculate that the impaired thymus organogenesis in chd7 morphants is partially attributed to impaired development of NCCs. The expression of migratory neural crest marker, crestin, was almost absent in chd7 morphant rhombomeres at $24 \mathrm{hpf}$ (Supplemental Figure S4). To further check the development of postmigratory NCCs, the expression of two post-migratory NCC markers was examined, hand 2 and $d l x 2 a$. Reduced expression of hand 2 was observed in the developing pharynx at $30 \mathrm{hpf}$, and the $d l x 2 a$ expression was specifically reduced in the population underneath the otic vesicle at $30 \mathrm{hpf}$, which would be segmented into pharyngeal arch 5 (Figure 4A). As pharyngeal segmentation continued, a distinct reduction in hand 2 expression may be observed in pharyngeal arches 4 and 5 and a prominent reduction may also be detected in the expression of $d l x 2 a$ in pharyngeal arch 4 in $c h d 7$ morphants at $48 \mathrm{hpf}$ (Figure 4B). These results demonstrated that not only the development of the third endodermal pouch but also the surrounding post-migratory neural crest cells in the fourth pharyngeal arch were severely impaired in chd7 morphants. 
A
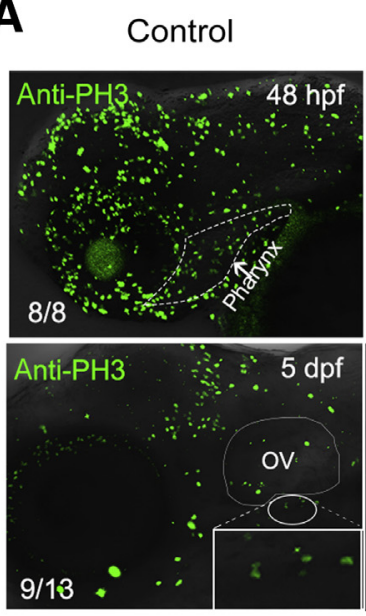

C
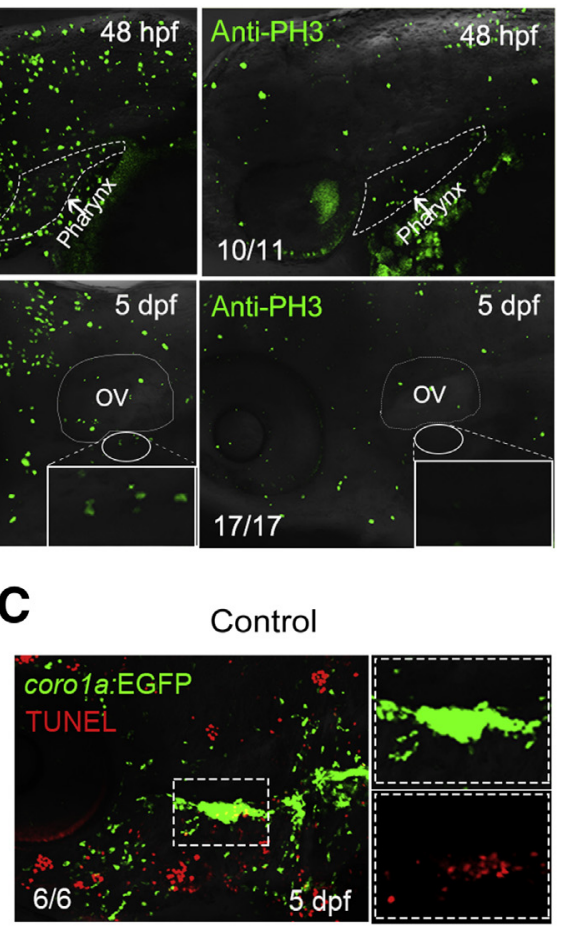

Anti-PH3

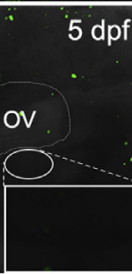

$17 / 17$

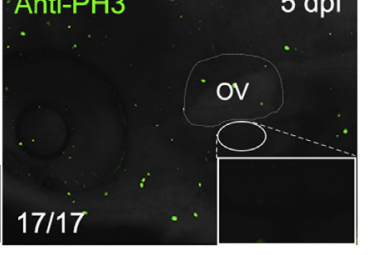

Control
B

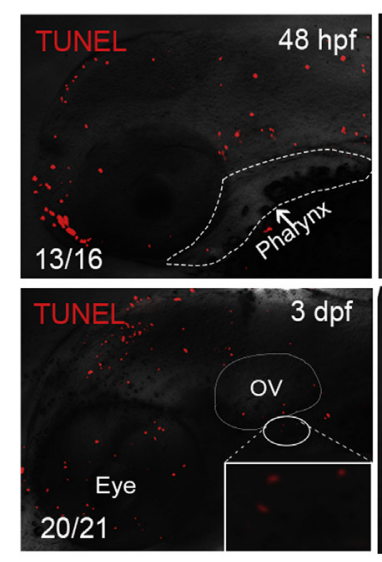

Control

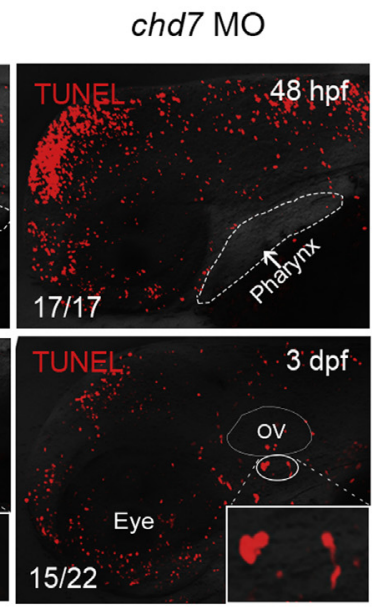

chd7 MO

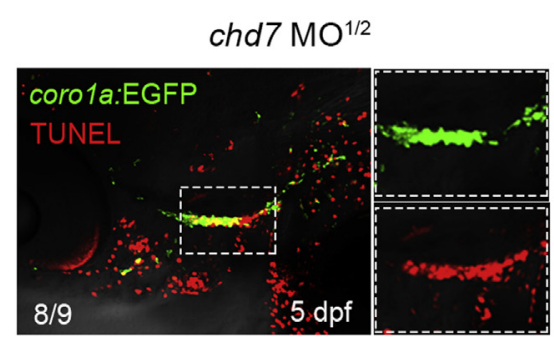

Figure 6 Reduced proliferation and increased apoptosis are present in chd7 morphants. A: Immunohistochemistry of anti-phospho-histone H3 (PH3) antibody in control and chd7 morphants at 48 and 5 days post-fertilization (dpf). White dashed lines indicate the pharynx and otic vesicle; white ellipses, the thymus; and insets, higher magnification of the thymus. B: Terminal dUTP nick end labeling (TUNEL) assay of the control and chd7 morphants at 48 and 3 dpf. White dashed lines indicate the regions of the pharynx and otic vesicle; white ellipses, the thymus; and insets, higher magnification of the thymus. C: TUNEL assay and immunostaining for corola:enhanced green fluorescence protein (EGFP) transgenic wild-type and chd7 morphants showing the apoptotic Tcell precursors in control and chd7 morphants at $5 \mathrm{dpf}$. White dashed rectangles denote the thymic regions. Insets denote higher magnification of the thymus. Original magnification: $\times 14(\mathbf{A}$ and $\mathbf{B}) ; \times 20$ (C). Hpf, hours post-fertilization; M0, morpholino; 0V, otic vesicle.

\section{Homing of Lymphoid Progenitors to the Thymic Rudiment Is Impaired in chd7 Morphants}

It has been demonstrated that the ability of thymus to attract the migratory lymphoid progenitors is crucial for commitment of lymphoid progenitors to early T-cell progenitors and subsequent differentiation, survival, and proliferation. In this study, T-cell and thymic development were severely impaired in $c h d 7$ morphants. It was examined whether the thymic homing was affected in $c h d 7$ morphants. The expression of a chemokine ligand, $c c l 25$, which was demonstrated to be indispensable for thymic homing and might be regulated by foxnl in mice and medaka, was first examined. ${ }^{32}$ Both qPCR and WISH data showed that $c c l 25 a$ and $c c l 25 b$ were down-regulated in the thymus of chd7 morphants (Figure 5, A and B, and Supplemental Figure S5), indicating that the ability of the thymus to attract lymphoid progenitors might be impaired. To further analyze dynamic trafficking of T-cell progenitors to the thymus in $c h d 7$ morphants, time-lapse imaging was used from 60 to $72 \mathrm{hpf}$. To distinguish lymphoid cells from myeloid cells, corola:EGFP/lyz:DsRed double transgenic embryos were used. The corola:EGFP ${ }^{+}$and lyz:DsRed ${ }^{-}$cells with round shape (lymphoid progenitors) were observed to migrate into the thymus of control embryos. However, a significantly reduced number of these cells migrated into the thymus of $\operatorname{ch} 77$ morphants (Figure 5, C and D, and Supplemental Video S1, S2, S3, and S4). These results indicated that the compromised ability of thymus to attract hematopoietic progenitors may also account for the reduced $\mathrm{T}$ cells in $c h d 7$ morphants.

\section{Reduced Proliferation and Increased Apoptosis Are Present in chd7 Morphants}

The defects of $\mathrm{T}$ lymphopoiesis and thymus organogenesis in $c h d 7$ morphants may be caused by impaired cell proliferation or abnormal apoptosis. Immunostaining with an anti-phospho-histone $\mathrm{H} 3$ antibody was first performed to detect proliferating cells in control and chd7 morphants. Phospho-histone H3-positive cells were significantly decreased in the region of the pharynx in chd7 morphants at $48 \mathrm{hpf}$, when thymus organogenesis was initiated, indicating that reduced proliferation might account for the impaired thymus organogenesis (Figure 6A). Moreover, at $5 \mathrm{dpf}$, when the thymus rudiment was formed and lymphoid progenitors were settled in it for subsequent development, the proliferating cells were decreased in the thymus region of chd7 morphants (Figure 6A). To evaluate apoptosis in $c h d 7$ morphants, the terminal dUTP nick end labeling assay was 

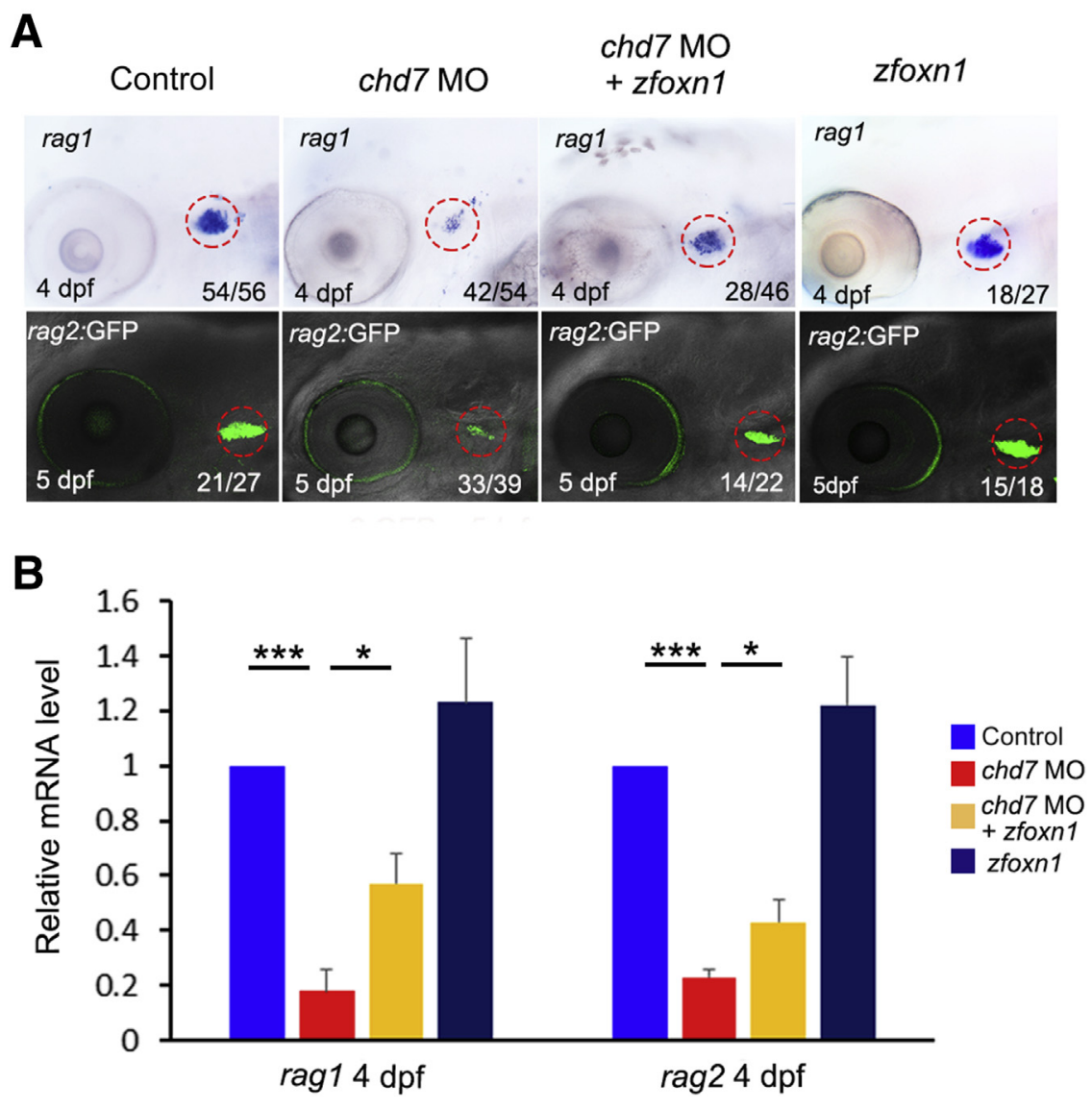

Figure 7 The expression of rag1 and rag2 can be rescued by foxn1 overexpression in chd7 morphants. A: Whole mount in situ hybridization (WISH) analysis for the expression of rag1 in control, chd7 morphant, chd7 morphants coinjected with foxn1 mRNA, and wild type injected with foxn 1 mRNA at 4 days post-fertilization (dpf) and WISH analysis for the expression of rag2 at 5 dpf. The dashed red circles indicate the regions of thymus. B: Quantitative RT-PCR analysis of the expression of rag1 and rag2 in control, chd7 morphants, chd7 morphants coinjected with zfoxn1 mRNA, and wild type injected with zfoxn1 mRNA at $4 \mathrm{dpf}$. Data are expressed as means \pm SD (B). ${ }^{*} P<0.05,{ }^{* * *} P<0.001$. Original magnification: $\times 15$ (A, WISH results); $\times 20$ (A, ISH results). GFP, green fluorescence protein; $M 0$, morpholino.

used. Apoptosis was not changed in the developing pharynx of $c h d 7$ morphants at $48 \mathrm{hpf}$, when thymus organogenesis was initiated and the neural crest cells had finished their migration and settled in the developing pharynx, indicating that the impaired organogenesis of the thymus might not be attributed to apoptosis but at least partially to compromised proliferation in chd7 morphants (Figure 6B). However, high numbers of apoptotic cells were detected in thymus area in chd7 morphants at $3 \mathrm{dpf}$, when thymus rudiment was formed and lymphoid progenitors were settled in it (Figure 6C). Furthermore, to find out whether these intrathymic apoptotic cells included T-cell precursors, a half dose of $c h d 7 \mathrm{MO}$ was injected into corola:EGFP embryos because a full dose of the MO severely compromised the homing of the lymphoid progenitors to thymus, and examined the apoptosis of intrathymic T-cell precursors. The cells dual labelled by terminal dUTP nick end labeling and GFP were much more than in the control group, indicating that increased apoptosis accounted for the reduction of corola-positive $\mathrm{T}$ cells in the thymus of chd7 morphants. Moreover, examination of apoptosis in the CHT region showed no change in apoptosis (data not shown) (Figure 6C). These results suggested that the reduction of T cells in $c h d 7$ morphants may be attributed to the impaired proliferation at early stage of thymus organogenesis and to the aberrant apoptosis in thymus at a later stage, particularly the apoptosis of T-cell precursors. In combination with the HSPC results, these data implied that the decrease of $\mathrm{T}$ cells in chd7 morphants may be mainly caused by the defects of thymus organogenesis.

\section{T-Cell Decrease in chd7 Morphants Can Be Partially Rescued by Forced Expression of foxn1}

Our results indicated that the down-regulated foxn1 might contribute to the impaired development of thymus in $c h d 7$ morphants. Many studies have documented that foxnl is crucial for TEC differentiation and maintenance. ${ }^{23,33,34}$ Furthermore, TEC regeneration can be achieved by upregulating foxn1 expression. ${ }^{35-37}$ These findings prompted us to speculate whether overexpression of foxnl may ameliorate the defects of thymus organogenesis and restore the reduced $\mathrm{T}$ cells in $c h d 7$ morphants. To test this idea, foxn1 mRNA and chd7 MO were coinjected into zebrafish embryos at the single-cell stage. More than half of the embryos survived in the foxnl overexpressing group (Supplemental Figure S6A). As anticipated, the expression of ragl was moderately restored at $4 \mathrm{dpf}$ (Figure 7A). Similarly, when the coinjection was performed in rag2:EGFP transgenic embryos, the number of GFPpositive T-cell precursors was also moderately restored (Figure 7A). The qPCR results of T-cell markers further 
A

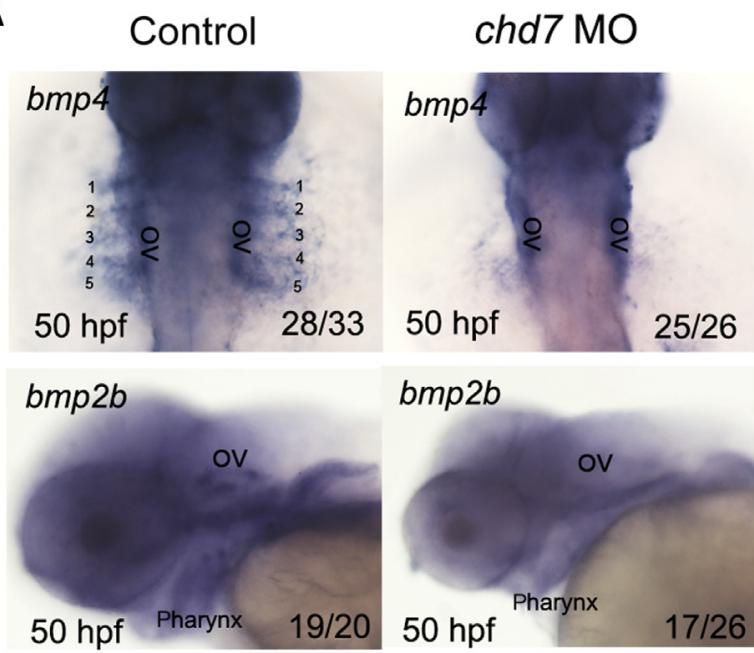

B Control
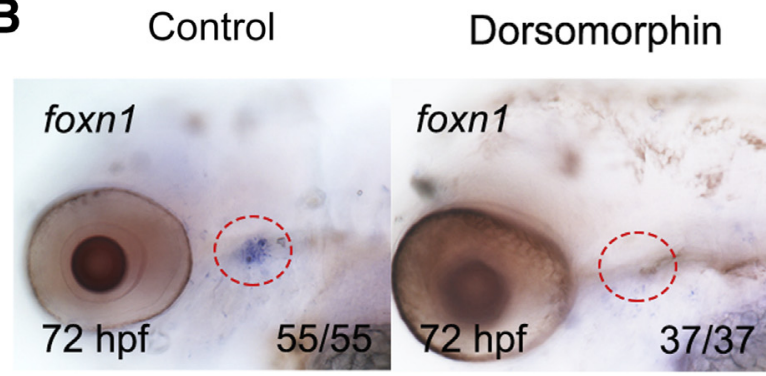

Figure 8 Chd7 regulates foxn1 expression in thymus organogenesis, probably mediated by bmp signaling. A: The expression of $b m p 4$ and $b m p 2 b$ in control and chd7 morphants at 50 hours post-fertilization (hpf). Numbers denote the pharyngeal arches. B: The expression of foxn1 in control and Dorsmorphin treatment group at $72 \mathrm{hpf}$. Dorsomorphin treatment was performed from 44 to $54 \mathrm{hpf}$. Red dashed circles indicate the thymus. Original magnification: $\times 12($ A) $; \times 15$ (B). M0, morpholino; OV, otic vesicle.

verified the rescue effects (Figure $7 \mathrm{~B}$ and Supplemental Figure 6B). Moreover, when foxnl was injected into wildtype embryos, the expression of ccl25a, a chemokine ligand important for the homing function of TECs, was largely restricted to the thymus area. Interestingly, ectopic expression was occasionally detected (Supplemental Figure S7), further demonstrating that foxn1 was critical for TEC differentiation and maintenance.

\section{Chd7 Regulates foxn1 Expression in Thymus}

Organogenesis, Which Is Probably Mediated by bmp Signaling

It has been well established that foxnl is crucial for thymus organogenesis; however, there is limited information regarding its upstream transcriptional regulation mechanism. Bmp4 has been found to be required for the establishment of thymic fate and foxn1 expression. ${ }^{38-40} \mathrm{Bmp} 2$ can be upregulated after deletion of Bmp4 to provide compensatory function in thymic organogenesis. ${ }^{41}$ It has been found that Chd7 might directly regulate Bmp4 expression in mouse. ${ }^{42}$
From these findings, it was conjectured that $C h d 7$ may regulate the process of thymus organogenesis through modulating the expression of Bmp 4 and foxn 1 in zebrafish. To verify this hypothesis, the expression of $b m p 4$ and $b m p 2 b$ was examined in $c h d 7$ morphants; the expression of bmp 4 was almost absent, whereas $b m p 2 b$ is reduced, in the pharynx at 50 and $48 \mathrm{hpf}$, respectively (Figure 8A), indicating that $c h d 7$ might regulate thymus organogenesis by modulating the expression of bmps. Moreover, Dorsomorphin was used to block the bmp signals from 44 to $54 \mathrm{hpf}$, a window of time during thymic organogenesis. foxn1 Transcript was strikingly reduced in the pharyngeal region at $60 \mathrm{hpf}$ (Figure 8B), indicating that the bmp signaling might regulate foxn 1 expression. Taken together, these results suggested that $\operatorname{chd} 7$ might regulate foxnl expression through bmp signaling in thymus organogenesis.

It has been demonstrated recently that foxn4, a metazoan ancestor of foxnl, can promote thymic epithelium formation in teleost fish. ${ }^{43}$ It was tested whether foxn 4 was involved in the thymic defects in $\operatorname{chd} 7$ morphants. The expression pattern of foxn 4 was examined. foxn 4 Was detected in the pharyngeal region at $4 \mathrm{dpf}$ but not 48 and $72 \mathrm{hpf}$, indicating that foxn 4 might not participate in the early thymic organogenesis (Supplemental Figure S8A). It was next checked whether the expression of foxn 4 changes in chd7 morphants at $4 \mathrm{dpf}$. Unexpectedly, little change of foxn 4 was detected in the pharyngeal region (Supplemental Figure S8B), indicating that foxn 4 might not be involved in the thymic defects in chd7 morphants. These results further suggested that the chd7-foxn1 molecular axis might be conserved between zebrafish and mammals.

\section{Chd7 Mutants and chd7 Morphants Display Similar Phenotypes}

To validate some of the phenotypes observed in spliceblockling MO treated embryos, a translation-blocking MO against chd7 (chd7 ATG-MO) was injected into the embryos at the single cell stage and the T-cell and TEC markers were also reduced in the thymus region at $4 \mathrm{dpf}$ (Supplemental Figure S9). Moreover, a $c h d 7$ mutation with a 23-bp deletion in the second exon was generated using zinc-finger nucleases (Figure 9A). The 23-bp deletion resulted in a deductive loss of all functional domains of Chd7 (Figure 9B) and was verified by genomic PCR (Figure 9C) and direct sequencing analysis (data not shown). The mutants displayed pericardial edema, cranial cartilage malformation, and swim bladder defects (Supplemental Figure S10), similar to the defects found in the morphants. Then, the expression of T-cell and thymic markers was examined in the mutants using WISH, and it was found that ikaros, il7r, foxn 1, ragl, and ccl25a were all down-regulated in the mutants at $4 \mathrm{dpf}$, indicating that $\mathrm{T}$ cell and thymic development were impaired in the absence of Chd7 (Figure 9D and Supplemental Figure S11), consistent with the observations in chd7 morphants. 
A

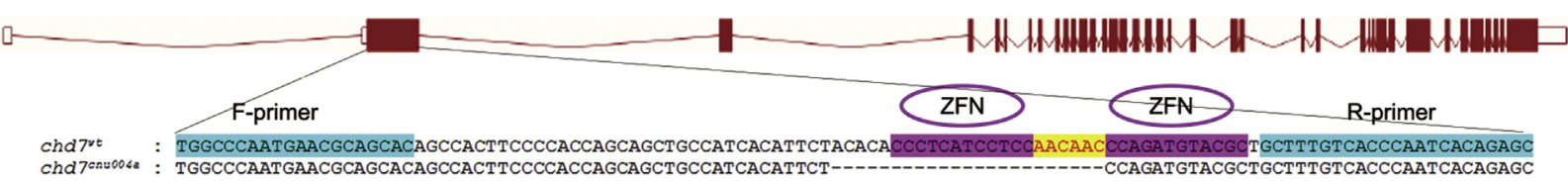

B
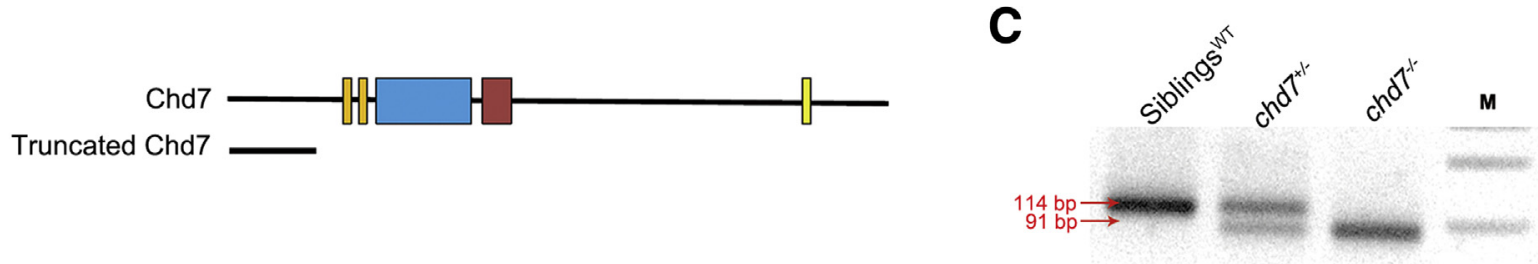

D

Offsprings of chd7 heterozygotes

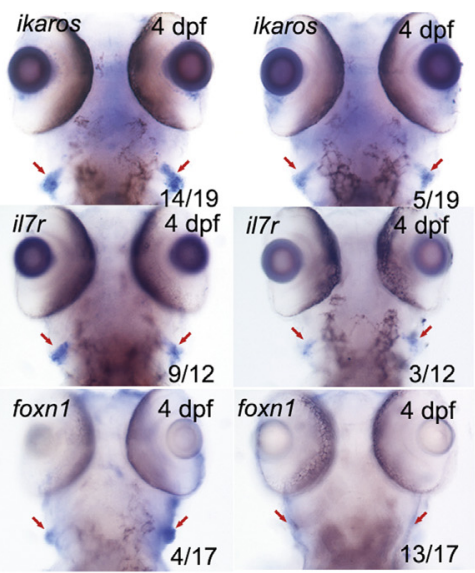

\section{E}

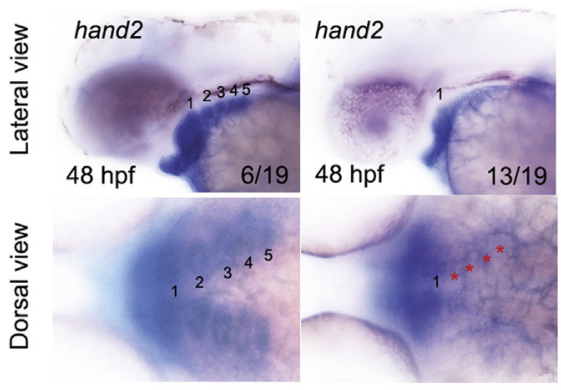

F Offsprings of $c h d 7$ heterozygotes

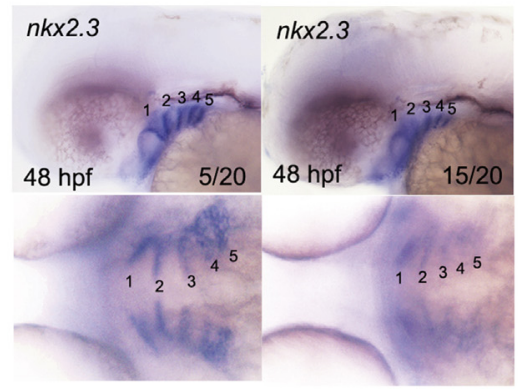

Figure 9 Chd7 mutants and chd7 morphants display similar phenotypes. A: Location and sequence of the zinc-finger nuclease (ZFN) target sites for the chd7 gene. B: Schematic of the zebrafish Chd7 protein in siblings and the mutants. Yellow, sapphire, brown, and yellow rectangles represent the chromodomain, SNF2, helicase, and BRK domains, respectively. C: Agarose gel electrophoresis analysis of wild-type siblings, chd7 heterozygote PCR products, and chd7 homozygote PCR products. M denotes the DNA marker. D: The expression of T-cell and thymic markers, ikaros, il7r, and foxn1, in the thymus in siblings and chd7 mutants at 4 days post-fertilization (dpf). Red arrows indicate the expression of T-cell and thymic markers in the thymus. E: The morphology of pharyngeal arches marked by expression of hand2 in siblings and chd7 mutants at 48 hours post-fertilization (hpf). Numbers denote the arches. Asterisks mark the defective arches. F: The morphology of pharyngeal endodermal pouches marked by expression of $n k \times 2.3$ in siblings and chd7 mutant at 48 hpf. Numbers denote the endodermal pouches. Original magnification: $\times 15(\mathbf{D}) ; \times 9(\mathbf{E}) ; \times 12(\mathbf{F})$. M0, morpholino.

Moreover, to determine whether the reduced expression of T-cell and thymic markers resulted from impaired thymic organogenesis, the development of the pharynx in the $\operatorname{chd} 7$ mutants was examined. The expression of endodermal marker, $n k x 2.3$, was down-regulated in all five pouches, and the expression of post-migratory marker, hand2, was dramatically down-regulated in arches $2,3,4$, and 5 at 48 hpf, which was consistent with the results in the $c h d 7$ morphants (Figure 9, E and F). These data demonstrated that interfering with $c h d 7$ expression at either genomic or transcriptional level resulted in similar T-cell and thymic developmental phenotypes.

\section{Discussion}

In this study, we demonstrate that the T-cell reduction in CHARGE syndrome can be phenocopied in zebrafish by knocking down or knocking out $c h d 7$, the main causative gene for the congenital disorder. T cells play a central role in cell-mediated immunity, and $\mathrm{T}$ lymphopenia usually leads to immunodeficiency. The T-cell numbers range from low to absent, and the severity of immunodeficiency varies from mild to severe among the reported individuals of CHARGE patients. In some cases, CHD7 is associated with severe combined immunodeficiency. ${ }^{8-11,13,14,44}$ The spectrum of immunodeficiency may be at least partially attributed to the different mutations found in $\mathrm{CHD7}$, ranging from benign and missense mutations to truncating mutations. Although truncating mutations seem to be associated with a more severe phenotype than missense variants, ${ }^{45}$ further studies are required to find out the correlation between genotype and phenotype of $\mathrm{CHD} 7$ mutations.

The defects of thymic organogenesis observed in chd7 morphants are consistent with the clinical reports of thymus hypoplasia or aplasia in CHARGE patients. It seems likely 
that the T lymphopenia and immunodeficiency in CHARGE syndrome mainly contributed to the defects of thymus organogenesis. However, the rate of reported thymus defects varies between different studies. Thymus hypoplasia or aplasia is rare in postnatal CHARGE syndrome cases, ${ }^{46}$ whereas it is observed in 7 of 10 CHARGE fetuses. ${ }^{15}$ This may be interpreted by, for example, the bias of the selected cohort of patients, the severity of various $\mathrm{CHD} 7$ mutations, and different imaging techniques used to detect the thymus. Otherwise, CHARGE patients with thymic defects are more prone to die from immunodeficiency or other accompanying anomalies, such as heart defects. It is also possible that the athymic patients are diagnosed as having DiGeorge syndrome rather than CHARGE syndrome. Furthermore, relatively normal morphology of the thymus might not necessarily mean normal function of the thymus or $\mathrm{T}$ cells in CHARGE patients because the homing function of the thymus might also be disrupted, as observed in our animal model.

The patients with proved variants in CHD7 are occasionally diagnosed as having complete DiGeorge syndrome, a typical athymic disorder that is mainly caused by defects in pharyngeal arches and pouches. ${ }^{47,48}$ To treat complete DiGeorge syndrome patients, transplantation of allogenic cultured thymic tissue was adopted as a therapy and obtained satisfying effects. ${ }^{49,50}$ Adoptive transfer of mature $\mathrm{T}$ cells from human leukocyte antigen identical siblings through bone marrow transplantation has been proposed. ${ }^{51}$ Nevertheless, no definite guidelines have been proposed for the clinical management of immunodeficiency in CHARGE syndrome. Various types of hematopoietic stem cells, including T-lymphocyte-depleted haploidentical paternal hematopoietic stem cells, have been used clinically but seem unsuccessful, ${ }^{10}$ which is probably attributable to a lack of understanding the underlying mechanisms. Our data highlight that the immunodeficiency in CHARGE syndrome is mainly attributed to thymic defects and indicate that restoring the morphology and function of thymus by, for example, thymic cell transplantation may be a potential strategy for treating immunologic abnormalities. However, the present study mainly focuses on the early development of T-cell and thymus organogenesis. Further studies are needed to clarify whether the thymic defects can recover with development and aging and whether the development of $\mathrm{T}$ cells is still compromised at later stages.

It is well established that Foxn1 plays a central role in thymus organogenesis and TEC differentiation. ${ }^{23,52}$ However, the upstream network that regulates the expression of Foxn1 still remains largely a mystery. ${ }^{52}$ In the present study, it was found that $c h d 7$ can regulate the expression of both foxn1 and bmp4. Several studies have demonstrated that Bmp4 regulates the expression of Foxn1 during the early development of thymus. And this role of Bmp4 is evolutionarily conserved between zebrafish and mammals. ${ }^{53}$ It has been demonstrated that $C h d 7$ might directly regulate the expression of Bmp4 in vitro, and Bmp4 is down-regulated in the forebrain of $C h d 7$-mutant mice. ${ }^{42}$ It has also been shown that Bmp4 participates in maintaining the expression of Foxn1 in mature thymus ${ }^{40}$ and Bmp4 is a critical component of a set of factors that direct the differentiation of human embryonic stem cells into functional thymic epithelial cells to support T-cell development. ${ }^{54}$ These studies are consistent with our finding that chd7 might regulate the expression of foxn1 in thymus organogenesis through modulating the bmp4 signaling. Besides participating in thymus morphogenesis, Bmp4 is also revealed to directly regulate the development of thymocytes by signaling through Bmp receptor expressed on the thymocytes. ${ }^{55}$ Further studies are required to clarify whether CHD7 regulates the expression of BMP4 in adults and whether BMP4 contributes to the T-cell lymphopenia in CHARGE syndrome by regulating the differentiation of thymic epithelial cells or the maturation of thymocytes into T cells or both.

A widely accepted pathogenetic theory of CHARGE syndrome is that it is caused by maldevelopment of the neural crest cells. ${ }^{31,56,57}$ However, the theory has often faced challenges because it could not explain all aspects of the disorder. Several other theories have been proposed [eg, a disruption of the mesenchymal-epithelial interaction (mesenchymal includes neural crest mesenchyme and mesoderm, and epithelial includes ectoderm and endoderm)]. ${ }^{56}$ Restoring $C h d 7$ expression specifically in neural crest cells fails to rescue the defects of pharyngeal arch artery in $C h d 7$ mutant mice, indicating that $C h d 7$ may also function in other tissues besides neural crest cells. ${ }^{58}$ Our study illustrates that $c h d 7$ is critical for the development of not only migratory (marked by crestin) and post-migratory neural crest cells (marked by crestin, dlx $2 a$, and hand2) but also the pharyngeal endoderm (marked by $n k x 2.3$ and $z n 5)$ and differentiated thymic epithelial cells (marked by foxn 1 and $c c l 25$ ) during thymus organogenesis. These data suggest that $\operatorname{chd} 7$ may participate in the interaction between epithelial and neural crest-derived mesenchymal cells in thymus morphogenesis. The down-regulation of bmp 4 in the pharynx of $c h d 7$ morphants further supports the idea because Bmp4 has been shown to mediate the mesenchymal-epithelial interaction during thymus development. ${ }^{39,59}$ The rescue of thymus defects in chd7 morphants by overexpressing foxn 1 also suggests the importance of endoderm-derived thymic epithelial cells in thymus development and $\mathrm{T}$ lymphopoiesis. Both our data and other studies support the mesenchymal-epithelial interaction theory in CHARGE pathogenesis. ${ }^{56}$

The increase of HSPCs peripherally in chd7-deficient embryos observed in this study is consistent with a previous report. ${ }^{60}$ Particularly, the increase of lymphoid precursors makes it difficult to explain the decrease of T cells in chd7 morphants; however, it can be easily explained by thymus defects. However, the contribution of the abnormal HSPCs cannot be excluded. For most patients, $\mathrm{T}$ lymphopenia is concomitant with T-cell dysfunction. Further investigation is required to examine whether the increased early HSPCs 
can develop normally into functional $\mathrm{T}$ cells and whether chd7 is involved in HSPCs at later stages. The developing thymocytes may cross talk with thymic stromal cells, and whether the abnormal HSPCs influence the development of thymus also needs further study. Although the report of T-cell decrease in CHARGE syndrome is usually accompanied with relatively normal B and natural killer cells, there are occasional reports of low or high numbers of these cells. ${ }^{12}$ A recent study demonstrates that $C h d 7$ deficiency might suppress leukemogenesis through inhibiting cellular proliferation. ${ }^{61}$ These reports suggest that the role of $C h d 7$ in hematopoiesis might be complex, and more study is needed to clarify whether abnormal HSPCs contribute to the rare abnormal $\mathrm{B}$ and natural killer cells and other potential hematopoietic defects. In addition, because CHARGE syndrome is occasionally diagnosed as DiGeorge syndrome because of their overlapping features, including immunodeficiency and T-cell decrease, the participation of CHD7 in HSPC development might be helpful for differential diagnosis and treatment of the two types of disorders.

In summary, we have illustrated the role of chd7 in early T-cell development and thymus organogenesis and the underlying molecular and cellular mechanisms. Our data indicate that, similar to complete DiGeorge syndrome, pharyngeal defects in arches and pouches, resulting in impaired thymic organogenesis, might be the major cause of thymic defects and T-cell decrease in CHARGE syndrome. From this, we propose that transplantation of allogenic cultured thymic tissue or adoptive transfer of mature $\mathrm{T}$ cells from human leukocyte antigen identical siblings through bone marrow transplantation should be used to treat severe combined immunodeficiency patients with CHARGE syndrome. Zebrafish will serve as an ideal model organism for studying immunologic deficiency in CHARGE syndrome.

\section{Acknowledgments}

We thank Wen-Ting Guo and Xin-Yi Zhao for technical help; Dr. Feng Liu for providing comments, critical reading, and providing the probes of ikaros, ragl, foxnl, and il7r; Drs. Lili Jing and Yan Zhang for invaluable comments and discussions; and the China Zebrafish Resource Center for providing the transgenic fishes.

\section{Supplemental Data}

Supplemental material for this article can be found at https://doi.org/10.1016/j.ajpath.2017.12.005.

\section{References}

1. Sanlaville D, Verloes A: CHARGE syndrome: an update. Eur J Hum Genet 2007, 15:389-399

2. Vissers LE, van Ravenswaaij CM, Admiraal R, Hurst JA, de Vries BB, Janssen IM, van der Vliet WA, Huys EH, de Jong PJ, Hamel BC, Schoenmakers EF, Brunner HG, Veltman JA, van
Kessel AG: Mutations in a new member of the chromodomain gene family cause CHARGE syndrome. Nat Genet 2004, 36:955-957

3. Blake KD, Hartshorne TS, Lawand C, Dailor AN, Thelin JW: Cranial nerve manifestations in CHARGE syndrome. Am J Med Genet A 2008, 146A:585-592

4. Chalouhi C, Faulcon P, Le Bihan C, Hertz-Pannier L, Bonfils P, Abadie V: Olfactory evaluation in children: application to the CHARGE syndrome. Pediatrics 2005, 116:e81-e88

5. Wang RY, Earl DL, Ruder RO, Graham JM Jr: Syndromic ear anomalies and renal ultrasounds. Pediatrics 2001, 108:E32

6. Zentner GE, Layman WS, Martin DM, Scacheri PC: Molecular and phenotypic aspects of CHD7 mutation in CHARGE syndrome. Am J Med Genet A 2010, 152A:674-686

7. Jongmans MC, Admiraal RJ, van der Donk KP, Vissers LE, Baas AF, Kapusta L, van Hagen JM, Donnai D, de Ravel TJ, Veltman JA, Geurts van Kessel A, De Vries BB, Brunner HG, Hoefsloot LH, van Ravenswaaij CM: CHARGE syndrome: the phenotypic spectrum of mutations in the CHD7 gene. J Med Genet 2006, 43:306-314

8. Chopra C, Baretto R, Duddridge M, Browning MJ: T-cell immunodeficiency in CHARGE syndrome. Acta Paediatr 2009, 98: $408-410$

9. Jyonouchi S, McDonald-McGinn DM, Bale S, Zackai EH, Sullivan KE: CHARGE (coloboma, heart defect, atresia choanae, retarded growth and development, genital hypoplasia, ear anomalies/deafness) syndrome and chromosome 22q11.2 deletion syndrome: a comparison of immunologic and nonimmunologic phenotypic features. Pediatrics 2009, 123:e871-e877

10. Gennery AR, Slatter MA, Rice J, Hoefsloot LH, Barge D, McLeanTooke A, Montgomery T, Goodship JA, Burt AD, Flood TJ, Abinun M, Cant AJ, Johnson D: Mutations in CHD7 in patients with CHARGE syndrome cause T-B + natural killer cell + severe combined immune deficiency and may cause Omenn-like syndrome. Clin Exp Immunol 2008, 153:75-80

11. Assing K, Nielsen C, Kirchhoff M, Madsen HO, Ryder LP, Fisker N $\mathrm{CD} 4+\mathrm{CD} 31+$ recent thymic emigrants in CHD7 haploinsufficiency (CHARGE syndrome): a case. Hum Immunol 2013, 74:1047-1050

12. Wong MT, Scholvinck EH, Lambeck AJ, van Ravenswaaij-Arts CM: CHARGE syndrome: a review of the immunological aspects. Eur J Hum Genet 2015, 23:1451-1459

13. Yu H, Zhang VW, Stray-Pedersen A, Hanson IC, Forbes LR, de la Morena MT, Chinn IK, Gorman E, Mendelsohn NJ, Pozos T, Wiszniewski W, Nicholas SK, Yates AB, Moore LE, Berge KE, Sorte H, Bayer DK, ALZahrani D, Geha RS, Feng Y, Wang G, Orange JS, Lupski JR, Wang J, Wong LJ: Rapid molecular diagnostics of severe primary immunodeficiency determined by using targeted next-generation sequencing. J Allergy Clin Immunol 2016 , 138:1142-1151.e2

14. Chinn IK, Shearer WT: Severe combined immunodeficiency disorders. Immunol Allergy Clin North Am 2015, 35:671-694

15. Sanlaville D, Etchevers HC, Gonzales M, Martinovic J, ClementZiza M, Delezoide AL, Aubry MC, Pelet A, Chemouny S, Cruaud C, Audollent S, Esculpavit C, Goudefroye G, Ozilou C, Fredouille C, Joye N, Morichon-Delvallez N, Dumez Y, Weissenbach J, Munnich A, Amiel J, Encha-Razavi F, Lyonnet S, Vekemans M, Attie-Bitach T: Phenotypic spectrum of CHARGE syndrome in fetuses with $\mathrm{CHD} 7$ truncating mutations correlates with expression during human development. J Med Genet 2006, 43:211-217

16. Thisse C, Thisse B: High-resolution in situ hybridization to wholemount zebrafish embryos. Nat Protoc 2008, 3:59-69

17. Chalasani SH, Sabol A, Xu H, Gyda MA, Rasband K, Granato M, Chien CB, Raper JA: Stromal cell-derived factor-1 antagonizes slit/robo signaling in vivo. J Neurosci 2007, 27:973-980

18. Langenau DM, Zon LI: The zebrafish: a new model of T-cell and thymic development. Nat Rev Immunol 2005, 5:307-317

19. Ma D, Wei Y, Liu F: Regulatory mechanisms of thymus and $\mathrm{T}$ cell development. Dev Comp Immunol 2013, 39:91-102 
20. Patten SA, Jacobs-McDaniels NL, Zaouter C, Drapeau P, Albertson RC, Moldovan F: Role of Chd7 in zebrafish: a model for CHARGE syndrome. PLoS One 2012, 7:e31650

21. Wang S, He Q, Ma D, Xue Y, Liu F: Irf4 regulates the choice between $\mathrm{T}$ lymphoid-primed progenitor and myeloid lineage fates during embryogenesis. Dev Cell 2015, 34:621-631

22. Li L, Yan B, Shi YQ, Zhang WQ, Wen ZL: Live imaging reveals differing roles of macrophages and neutrophils during zebrafish tail fin regeneration. J Biol Chem 2012, 287:25353-25360

23. Vaidya HJ, Briones Leon A, Blackburn CC: FOXN1 in thymus organogenesis and development. Eur J Immunol 2016, 46:1826-1837

24. Gordon J, Wilson VA, Blair NF, Sheridan J, Farley A, Wilson L, Manley NR, Blackburn CC: Functional evidence for a single endodermal origin for the thymic epithelium. Nat Immunol 2004, 5:546-553

25. Lee KH, Xu Q, Breitbart RE: A new tinman-related gene, nkx2.7, anticipates the expression of $\mathrm{nkx} 2.5$ and nkx2.3 in zebrafish heart and pharyngeal endoderm. Dev Biol 1996, 180:722-731

26. Crump JG, Swartz ME, Kimmel CB: An integrin-dependent role of pouch endoderm in hyoid cartilage development. PLoS Biol 2004, 2:E244

27. Piotrowski T, Nusslein-Volhard C: The endoderm plays an important role in patterning the segmented pharyngeal region in zebrafish (Danio rerio). Dev Biol 2000, 225:339-356

28. Griffith AV, Cardenas K, Carter C, Gordon J, Iberg A, Engleka K, Epstein JA, Manley NR, Richie ER: Increased thymus- and decreased parathyroid-fated organ domains in Splotch mutant embryos. Dev Biol 2009, 327:216-227

29. Jenkinson WE, Bacon A, White AJ, Anderson G, Jenkinson EJ: An epithelial progenitor pool regulates thymus growth. J Immunol 2008, 181:6101-6108

30. Chen L, Zhao P, Wells L, Amemiya CT, Condie BG, Manley NR: Mouse and zebrafish Hoxa3 orthologues have nonequivalent in vivo protein function. Proc Natl Acad Sci U S A 2010, 107:10555-10560

31. Bajpai R, Chen DA, Rada-Iglesias A, Zhang J, Xiong Y, Helms J, Chang CP, Zhao Y, Swigut T, Wysocka J: CHD7 cooperates with PBAF to control multipotent neural crest formation. Nature 2010, 463:958-962

32. Bajoghli B, Aghaallaei N, Hess I, Rode I, Netuschil N, Tay BH, Venkatesh B, Yu JK, Kaltenbach SL, Holland ND, Diekhoff D, Happe C, Schorpp M, Boehm T: Evolution of genetic networks underlying the emergence of thymopoiesis in vertebrates. Cell 2009, 138:186-197

33. Nowell CS, Bredenkamp N, Tetelin S, Jin X, Tischner C, Vaidya H, Sheridan JM, Stenhouse FH, Heussen R, Smith AJ, Blackburn CC: Foxn 1 regulates lineage progression in cortical and medullary thymic epithelial cells but is dispensable for medullary sublineage divergence. PLoS Genet 2011, 7:e1002348

34. Romano R, Palamaro L, Fusco A, Giardino G, Gallo V, Del Vecchio L, Pignata C: FOXN1: a master regulator gene of thymic epithelial development program. Front Immunol 2013, 4:187

35. Bredenkamp N, Nowell CS, Blackburn CC: Regeneration of the aged thymus by a single transcription factor. Development 2014, 141: $1627-1637$

36. Zook EC, Krishack PA, Zhang S, Zeleznik-Le NJ, Firulli AB, Witte PL, Le PT: Overexpression of Foxn1 attenuates age-associated thymic involution and prevents the expansion of peripheral CD4 memory T cells. Blood 2011, 118:5723-5731

37. Bredenkamp N, Ulyanchenko S, O'Neill KE, Manley NR, Vaidya HJ, Blackburn CC: An organized and functional thymus generated from FOXN1-reprogrammed fibroblasts. Nat Cell Biol 2014, 16:902-908

38. Patel SR, Gordon J, Mahbub F, Blackburn CC, Manley NR: Bmp4 and Noggin expression during early thymus and parathyroid organogenesis. Gene Expr Patterns 2006, 6:794-799

39. Neves H, Dupin E, Parreira L, Le Douarin NM: Modulation of Bmp4 signalling in the epithelial-mesenchymal interactions that take place in early thymus and parathyroid development in avian embryos. Dev Biol 2012, 361:208-219

40. Barsanti M, Lim JM, Hun ML, Lister N, Wong K, Hammett MV, Lepletier A, Boyd RL, Giudice A, Chidgey AP: A novel
Foxn1eGFP/+ mouse model identifies Bmp4-induced maintenance of Foxn1 expression and thymic epithelial progenitor populations. Eur J Immunol 2017, 47:291-304

41. Bleul CC, Boehm T: BMP signaling is required for normal thymus development. J Immunol 2005, 175:5213-5221

42. Jiang X, Zhou Y, Xian L, Chen W, Wu H, Gao X: The mutation in Chd7 causes misexpression of Bmp4 and developmental defects in telencephalic midline. Am J Pathol 2012, 181:626-641

43. Swann JB, Weyn A, Nagakubo D, Bleul CC, Toyoda A, Happe C, Netuschil N, Hess I, Haas-Assenbaum A, Taniguchi Y, Schorpp M, Boehm T: Conversion of the thymus into a bipotent lymphoid organ by replacement of FOXN1 with its paralog, FOXN4. Cell Rep 2014, 8:1184-1197

44. Boudny P, Kurrer MO, Stamm B, Laeng RH: Malakoplakia of the colon in an infant with severe combined immunodeficiency (SCID) and charge association. Pathol Res Pract 2000, 196:577-582

45. Bergman JE, Janssen N, van der Sloot AM, de Walle HE, Schoots J, Rendtorff ND, Tranebjaerg L, Hoefsloot LH, van RavenswaaijArts CM, Hofstra RM: A novel classification system to predict the pathogenic effects of CHD7 missense variants in CHARGE syndrome. Hum Mutat 2012, 33:1251-1260

46. Blake KD, Davenport SLH, Hall BD, Hefner MA, Pagon RA, Williams MS, Lin AE, Graham JM: CHARGE association: an update and review for the primary pediatrician. Clin Pediatr 1998, 37: $159-173$

47. Markert ML, Devlin BH, Chinn IK, McCarthy EA: Thymus transplantation in complete DiGeorge anomaly. Immunol Res 2009, 44: $61-70$

48. Janda A, Sedlacek P, Mejstrikova E, Zdrahalova K, Hrusak O, Kalina T, Sieglova Z, Zizkova H, Formankova R, Keslova P, Hubacek P, Sediva A, Bartunkova J, Dlask K, Stary J: Unrelated partially matched lymphocyte infusions in a patient with complete DiGeorge/CHARGE syndrome. Pediatr Transplant 2007, 11: 441-447

49. Rice HE, Skinner MA, Mahaffey SM, Oldham KT, Ing RJ, Hale LP, Markert ML: Thymic transplantation for complete DiGeorge syndrome: medical and surgical considerations. J Pediatr Surg 2004, 39: $1607-1615$

50. Markert ML, Boeck A, Hale LP, Kloster AL, McLaughlin TM, Batchvarova MN, Douek DC, Koup RA, Kostyu DD, Ward FE, Rice HE, Mahaffey SM, Schiff SE, Buckley RH, Haynes BF: Transplantation of thymus tissue in complete DiGeorge syndrome. N Engl J Med 1999, 341:1180-1189

51. McGhee SA, Lloret MG, Stiehm ER: Immunologic reconstitution in 22q deletion (DiGeorge) syndrome. Immunol Res 2009, 45: $37-45$

52. Gordon J, Manley NR: Mechanisms of thymus organogenesis and morphogenesis. Development 2011, 138:3865-3878

53. Soza-Ried C, Bleul CC, Schorpp M, Boehm T: Maintenance of thymic epithelial phenotype requires extrinsic signals in mouse and zebrafish. J Immunol 2008, 181:5272-5277

54. Parent AV, Russ HA, Khan IS, LaFlam TN, Metzger TC, Anderson MS, Hebrok M: Generation of functional thymic epithelium from human embryonic stem cells that supports host $\mathrm{T}$ cell development. Cell Stem Cell 2013, 13:219-229

55. Hager-Theodorides AL, Ross SE, Sahni H, Mishina Y, Furmanski AL, Crompton T: Direct BMP2/4 signaling through BMP receptor IA regulates fetal thymocyte progenitor homeostasis and differentiation to CD4+CD8+ double-positive cell. Cell Cycle 2014, 13:324-333

56. Williams MS: Speculations on the pathogenesis of CHARGE syndrome. Am J Med Genet A 2005, 133A:318-325

57. Siebert JR, Graham JM Jr, MacDonald C: Pathologic features of the CHARGE association: support for involvement of the neural crest. Teratology 1985, 31:331-336

58. Randall V, McCue K, Roberts C, Kyriakopoulou V, Beddow S, Barrett AN, Vitelli F, Prescott K, Shaw-Smith C, Devriendt K, 
Bosman E, Steffes G, Steel KP, Simrick S, Basson MA Illingworth E, Scambler PJ: Great vessel development requires biallelic expression of Chd7 and Tbx1 in pharyngeal ectoderm in mice. J Clin Invest 2009, 119:3301-3310

59. Gordon J, Patel SR, Mishina Y, Manley NR: Evidence for an early role for BMP4 signaling in thymus and parathyroid morphogenesis. Dev Biol 2010, 339:141-154
60. Huang HT, Kathrein KL, Barton A, Gitlin Z, Huang YH, Ward TP, Hofmann O, Dibiase A, Song A, Tyekucheva S, Hide W, Zhou Y, Zon LI: A network of epigenetic regulators guides developmental haematopoiesis in vivo. Nat Cell Biol 2013, 15:1516-1525

61. Zhen T, Kwon E, Zhao L, Hsu J, Hyde RK, Lu Y, Alemu L, Speck NA, Liu PP: CHD7 deficiency delays leukemogenesis in mice induced by Cbfb-MYH11. Blood 2017, 130:2431-2442 